\title{
Stability of Lewis and Vogel's result
}

\section{David Preiss and Tatiana Toro}

\begin{abstract}
Lewis and Vogel proved that a bounded domain whose Poisson kernel is constant and whose surface measure to the boundary has at most Euclidean growth is a ball. In this paper we show that this result is stable under small perturbations. In particular a bounded domain whose Poisson kernel is smooth and close to a constant, and whose surface measure to the boundary has at most Euclidean growth is a smooth deformation of a ball.
\end{abstract}

\section{Introduction}

Lewis and Vogel proved (see [10], [11]) that a bounded domain whose harmonic measure (with respect to a fixed point) is a constant multiple of the surface measure to the boundary (i.e. a domain whose Poisson kernel is constant) is a ball, provided the surface measure has at most Euclidean growth. In this paper we prove that this result is stable under small perturbations. Namely a bounded domain whose Poisson kernel is almost constant, and whose surface measure to the boundary has at most Euclidean growth, is geometrically close to a ball.

Both of these results can be viewed as free boundary regularity results for the Poisson kernel. An interesting feature is that regularity of the free boundary is proved without an a-priori assumption of flatness. In fact, our main theorem states that a domain whose Poisson kernel is almost constant has a locally flat boundary (see Theorem 2.1). Once the boundary is known to be locally flat the proof of regularity is standard.

Let $\Omega \subset \mathbb{R}^{n+1}$ be a bounded domain and a set of locally finite perimeter such that $0 \in \Omega$ and $\mathcal{H}^{n}(\partial \Omega)<\infty$. Let $\omega$ denote the harmonic measure of $\Omega$ with pole at 0 . Let $\sigma$ denote the surface measure of the boundary, i.e. $\sigma=\mathcal{H}^{n}\left\llcorner\partial \Omega\right.$. Let $h=\frac{d \omega}{d \sigma}$ denote the Poisson kernel of $\Omega$ with pole at 0 .

2000 Mathematics Subject Classification: 28A75, 31A20, 35R35.

Keywords: Harmonic measure, Poisson kernel, Reifenberg flat. 
First we state Lewis and Vogel's result. Then we state one of our results which emphasizes the stability of their result.

Theorem 1.1 ([10]) Assume that $\Omega \subset \mathbb{R}^{n+1}$ satisfies

$$
\begin{gathered}
\sup _{0<r<1} \sup _{Q \in \partial \Omega} \frac{\mathcal{H}^{n}(B(Q, r) \cap \partial \Omega)}{r^{n}}<\infty, \\
\omega=\mathcal{H}^{n}\llcorner\partial \Omega .
\end{gathered}
$$

Then $\Omega$ is a ball of center 0 and radius $R>0$ such that $\mathcal{H}^{n}(\partial B(0, R))=1$.

Theorem 1.2 Assume that $\Omega \subset \mathbb{R}^{n+1}$ satisfies

$$
\sup _{0<r<1} \sup _{Q \in \partial \Omega} \frac{\mathcal{H}^{n}(B(Q, r) \cap \partial \Omega)}{r^{n}}<\infty
$$

$$
\frac{d \omega}{d \sigma}=h \quad \text { and } \quad \sup _{\partial \Omega}|\log h|<\varepsilon,
$$

for some $\varepsilon>0$ small enough.

Then $\Omega$ is a "smooth" deformation of $B(0, R)$ and $D[B(0, R), \Omega]<4 \varepsilon$. Here $\mathcal{H}^{n}(\partial B(0, R))=1$ and $D$ denotes the Hausdorff distance.

The paper is organized as follows: in section 2 we introduce some definitions and state the main theorem precisely. In section 3 we prove that the gradient of the Green function near the boundary is controlled by the Poisson kernel. This is a consequence of the fact that the gradient of the Green function is a subharmonic function on a bounded domain and therefore the values near the boundary are controlled by the boundary values. Recall that the Poisson kernel is basically the derivative of the Green function at the boundary. As a consequence we show that if $\Omega$ satisfies (1.3) and (1.4) then $D[B(0, R), \Omega]<4 \varepsilon$. In section 4 we introduce a local notion of flatness which involves the geometry of the boundary at a point and the behavior of $G$ and $\log h$ near that point. This allows us to show that $\partial \Omega$ is locally flat. In section 5 we present some applications of Theorem 2.1.

Acknowledgments: The authors would like to thank B. Kirchheim for his interest in the problem as well as for very stimulating conversations 


\section{Preliminaries}

In this section we introduce the definitions needed to state our main results. The main theorem appears at the end of the section and it is proved in section 4 . We always assume that $n \geq 2$.

Definition 2.1 Let $\Sigma \subset \mathbb{R}^{n+1}$ be a locally compact set, and let $\delta>0$. We say that $\Sigma$ is $\delta$-Reifenberg flat if for each compact set $K \subset \mathbb{R}^{n+1}$, there exists $R_{K}>0$ such that for every $Q \in K \cap \Sigma$ and every $R \in\left(0, R_{K}\right]$ there exists an $n$-dimensional plane $L(Q, r)$ containing $Q$ such that

$$
\frac{1}{r} D[\Sigma \cap B(Q, r), L(Q, r) \cap B(Q, r)] \leq \delta .
$$

Here $B(Q, r)$ denotes the $(n+1)$-dimensional ball of radius $r$ and center $Q$, and $D$ denotes the Hausdorff distance.

Recall that for $A, B \subset \mathbb{R}^{n+1}$,

$$
D[A, B]=\sup \{d(a, B): a \in A\}+\sup \{d(b, A): b \in B\} .
$$

Note that the previous definition is only significant for $\delta>0$ small. We denote by

$$
\theta(Q, r)=\inf _{L}\left\{\frac{1}{r} D[\Sigma \cap B(Q, r), L \cap B(Q, r)]\right\},
$$

where the infimum is taken over all $n$-planes containing $Q$.

Definition 2.2 Let $\Omega \subset \mathbb{R}^{n+1}$ be a set of locally finite perimeter (see [2]), $\partial \Omega$ is said to be Ahlfors regular if the surface measure to the boundary, i.e., the restriction of the $n$-dimensional Hausdorff measure to $\partial \Omega, \sigma=\mathcal{H}^{n}\llcorner\partial \Omega$, is Ahlfors regular. That is there exists a constant $C>1$ so that for $Q \in \partial \Omega$ and $r \in(0, \operatorname{diam} \Omega)$

$$
C^{-1} r^{n} \leq \sigma(B(Q, r)) \leq C r^{n} .
$$

Definition 2.3 Let $\Omega \subset \mathbb{R}^{n+1}$ be a bounded set. We say that $\Omega$ has the separation property if there exists $R>0$ such that for $Q \in \partial \Omega$ and $r \in(0, R]$ there exists an $n$-dimensional plane $\mathcal{L}(Q, r)$ containing $Q$ and a choice of unit normal vector to $\mathcal{L}(Q, r), \overrightarrow{n_{Q, r}}$ satisfying

$$
\mathcal{T}^{+}(Q, r)=\left\{X=(x, t)=x+t \overrightarrow{n_{Q, r}} \in B(Q, r): x \in \mathcal{L}(Q, r), t>\frac{r}{4}\right\} \subset \Omega,
$$

and

$\mathcal{T}^{-}(Q, r)=\left\{X=(x, t)=x+t \overrightarrow{n_{Q, r}} \in B(Q, r): x \in \mathcal{L}(Q, r), t<-\frac{r}{4}\right\} \subset \Omega^{c}$. 
The notation $(x, t)=x+t \overrightarrow{n_{Q, r}}$ is used to denote a point in $\mathbb{R}^{n+1}$. The first component, $x$, of the pair belongs to an $n$-dimensional affine space whose unit normal vector is $\overrightarrow{n_{Q, r}}$. The second component $t$ belongs to $\mathbb{R}$. From the context it will always be clear what affine hyperplane $x$ belongs to, and what the orientation of the unit normal vector is.

Definition 2.4 Let $\delta \in\left(0, \delta_{n}\right)$, where $\delta_{n}$ is chosen appropriately (see note below) and let $\Omega \subset \mathbb{R}^{n+1}$. We say that $\Omega$ is a $\delta$-Reifenberg flat domain or a Reifenberg flat domain if $\Omega$ has the separation property and $\partial \Omega$ is $\delta$-Reifenberg flat.

When we consider $\delta$-Reifenberg flat domains in $\mathbb{R}^{n+1}$ we assume that $\delta_{n}>0$ is small enough, in order to ensure that we are working on NTA domains (see definition in [5] and also [7, Theorem 3.1]).

Definition 2.5 A set of locally finite perimeter $\Omega \subset \mathbb{R}^{n+1}$ is said to be a chord arc domain, if $\Omega$ is an NTA domain whose boundary is Ahlfors regular.

Definition 2.6 Let $\delta \in\left(0, \delta_{n}\right)$. A set of locally finite perimeter $\Omega \subset \mathbb{R}^{n+1}$ is said to be a $\delta$-Reifenberg flat chord arc domain, if $\Omega$ is a $\delta$-Reifenberg flat domain whose boundary is Ahlfors regular.

Definition 2.7 Let $\delta \in\left(0, \delta_{n}\right)$. A bounded set of locally finite perimeter $\Omega$ is said to be a $\delta$-chord arc domain or a chord arc domain with small constant if $\Omega$ is a $\delta$-Reifenberg flat domain, $\partial \Omega$ is Ahlfors regular and there exists $R>0$ so that

$$
\sup _{Q \in \partial \Omega}\|\vec{n}\|_{*}(Q, R)<\delta
$$

Here $\vec{n}$ denotes the unit normal vector to the boundary,

$$
\|\vec{n}\|_{*}(Q, R)=\sup _{0<s<R}\left(f_{B(Q, s)}\left|\vec{n}-\overrightarrow{n_{Q, s}}\right|^{2} d \sigma\right)^{\frac{1}{2}}
$$

and $\overrightarrow{n_{Q, s}}=\oint_{B(Q, s)} \vec{n} d \sigma$

Definition 2.8 Let $\Omega \subset \mathbb{R}^{n+1}$ be a chord arc domain. Let $f \in L_{\text {loc }}^{2}(d \sigma)$, we say that $f \in \operatorname{BMO}(\partial \Omega)$ if

$$
\|f\|_{*}=\sup _{r>0} \sup _{Q \in \partial \Omega}\left(f_{B(Q, r)}\left|f-f_{Q, r}\right|^{2} d \sigma\right)^{\frac{1}{2}}<\infty .
$$

Here $f_{Q, r}=f_{B(Q, r)} f d \sigma$, and $\sigma=\mathcal{H}^{n}\llcorner\partial \Omega$. 
Definition 2.9 Let $\Omega \subset \mathbb{R}^{n+1}$ be a chord arc domain. We denote by $\operatorname{VMO}(\partial \Omega)$ the closure in $\mathrm{BMO}(\partial \Omega)$ of the set of uniformly continuous bounded functions defined on $\partial \Omega$.

From now on we assume that $\Omega \subset \mathbb{R}^{n+1}$ is a bounded domain and a set of locally finite perimeter such that $0 \in \Omega$ and $\mathcal{H}^{n}(\partial \Omega)<\infty$. Let $\omega$ denote the harmonic measure of $\Omega$ with pole at 0 . Let $\sigma$ denote the surface measure of the boundary. Let $h=\frac{d \omega}{d \sigma}$ denote the Poisson kernel of $\Omega$ with pole at 0 .

Theorem 2.1 Assume that $\Omega \subset \mathbb{R}^{n+1}$ satisfies

$$
\sup _{0<r<1} \sup _{Q \in \partial \Omega} \frac{\mathcal{H}^{n}(B(Q, r) \cap \partial \Omega)}{r^{n}}<\infty .
$$

Then given $\delta>0$ small enough there exists $\varepsilon>0$ such that if

$$
\sup _{\partial \Omega}|\log h|<\varepsilon
$$

then $\partial \Omega$ is $\delta$-Reifenberg flat.

\section{Rough geometric properties}

The Main Lemma below provides a crucial estimate for the gradient of the Green function near the boundary in terms of the Poisson kernel. It allows us to deduce that under the hypothesis of Theorem 2.1, $\partial \Omega$ is contained in a very thin annular region.

Main Lemma Let $\Omega \subset \mathbb{R}^{n+1}, 0 \in \Omega$. Let $G$ denote the Green function of $\Omega$ with pole 0 and let $h$ be the corresponding Poisson kernel. Assume that

$$
\sup _{0<r<1} \sup _{Q \in \partial \Omega} \frac{\mathcal{H}^{n}(B(Q, r) \cap \partial \Omega)}{r^{n}}<\infty
$$

and

$$
\sup _{\partial \Omega}|\log h|<\varepsilon
$$

for some $\varepsilon \in(0,1)$. Then

$$
\limsup _{X \rightarrow P}|\nabla G(X)| \leq e^{\varepsilon} \quad \forall P \in \partial \Omega .
$$

Let

$$
K_{0}=\sup _{0<r<1} \sup _{Q \in \partial \Omega} \frac{\mathcal{H}^{n}(B(Q, r) \cap \partial \Omega)}{r^{n}}<\infty
$$


Lemma 3.1 Under the assumptions above, let $R>0$ be such that $B(0, R) \subset$ $\Omega$ and $\partial B(0, R) \cap \partial \Omega \neq \emptyset$. Then

$$
|\nabla G(X)| \leq C_{n} K_{0} \quad \forall X \in \Omega \text { such that } d(X) \leq \frac{R}{2} .
$$

Here $d(X)$ denotes the distance from $X$ to $\partial \Omega$.

Note that (3.5) and the maximum principle for harmonic functions yield

$$
|\nabla G(X)| \leq C\left(n, K_{0}, R\right) \quad \forall X \in \Omega \backslash B\left(0, \frac{R}{2}\right) .
$$

Proof. Apply the Riesz decomposition theorem for subharmonic functions to $G$ (see [4, Theorem 6.18]). Let $Q \in \partial \Omega$ be such that $0 \notin B(Q, r)$

$$
\begin{aligned}
G(Q)= & \int_{\partial B(Q, r)} G(Z) d \sigma(Z) \\
& -\frac{1}{(n-1)(n+1) \omega_{n+1}} \int_{B(Q, r) \cap \partial \Omega}\left(\frac{1}{|Z-Q|^{n-1}}-\frac{1}{r^{n-1}}\right) d \omega(Z) .
\end{aligned}
$$

Using Fubini and the fact that $G(Q)=0,(3.7)$ yields

$$
f_{\partial B(Q, r)} G(Z) d \sigma(Z)=\frac{1}{(n+1) \omega_{n+1}} \int_{0}^{r} \frac{\omega(B(Q, t))}{t^{n}} d t .
$$

Note that (2.10) and (3.4) imply that for $t<1$,

$$
\omega(B(Q, t)) \leq e^{\varepsilon} \mathcal{H}^{n}(B(Q, t) \cap \partial \Omega) \leq e^{\varepsilon} K_{0} t^{n} .
$$

Combining (3.8) and (3.9) we have that for $\varepsilon<1$

$$
\oint_{\partial B(Q, r)} G(Z) d \sigma(Z) \leq C_{n} K_{0} r
$$

whenever $Q \in \partial \Omega$ and $0 \notin B(Q, r)$.

Let $X \in \Omega$ such that $d(X) \leq \frac{R}{2}$, there exists $Q \in \partial \Omega$ such that $d(X)=$ $r=|X-Q|$. Since $r<\frac{R}{2}$ then $0 \notin B(Q, 2 r)$, and the representation formula for subharmonic functions implies

$$
G(X) \leq \frac{(2 r)^{2}-|X-Q|^{2}}{(n+1) \omega_{n+1}(2 r)} \int_{\partial B(Q, 2 r)} \frac{G(Z)}{|Z-X|^{n+1}} d \sigma(Z) .
$$

Since $|Z-X| \geq r$ for $X \in \partial B(Q, r),(3.10)$ and (3.11) yield

$$
G(X) \leq \frac{3}{2} \int_{\partial B(Q, 2 r)} G(Z) d \sigma(Z) \leq C_{n} K_{0} r=C_{n} K_{0} d(X) .
$$


Standard estimates for harmonic functions on $\Omega \backslash B\left(0, \frac{R}{2}\right)$ ensure that

$$
|\nabla G(X)| \leq C_{n} \frac{G(X)}{d(X)}=C_{n} K_{0}
$$

The proof of the Main Lemma is a slight variation of the proof that appears in [10]. We sketch the proof and try to indicate as we go along what the ideas behind the calculations are. For further details we refer the reader to [10] and [11].

Proof of Main Lemma: Let $M=\limsup _{X \rightarrow \partial \Omega}|\nabla G(X)|$. Assume that $M>e^{\varepsilon}$. Let $\delta \in\left(0,10^{-10}\right)$ and let $X_{0} \in \Omega$ be such that $d\left(X_{0}\right) \leq \frac{R}{4}$ and

$$
\left|\nabla G\left(X_{0}\right)\right| \geq M-\delta
$$

Let $W(X)=\max \{|\nabla G(X)|-(M-2 \delta) ; 0\}$, observe that $W\left(X_{0}\right) \geq \delta$, and that $W$ is subharmonic in $\Omega \backslash B\left(0, \frac{R}{2}\right)$. Let $G_{0}$ be the Green's function of $\Omega$ with pole at $X_{0}$. By Sard's theorem we can choose $t>0$ such that $\left|\nabla G_{0}(X)\right| \neq 0$ on $\left\{X: G_{0}(X)=t\right\}$. Green's second identity, the fact that $W$ is subharmonic on $\Omega \backslash B\left(0, \frac{R}{2}\right)$, the maximum principle applied to $G$ and $G_{0}$ on $\Omega \backslash B\left(0, \frac{R}{2}\right)$ and $\Omega \backslash B\left(X_{0}, \frac{d_{0}}{2}\right)$ respectively, where $d_{0}=d\left(X_{0}\right)$ and (3.5) yield

$$
\frac{1}{6} \leq \int_{\{|\nabla G|>M-2 \delta\} \cap\left\{G_{0}=t\right\}} \frac{\partial G_{0}}{\partial \nu}(Y) d \mathcal{H}^{n}(Y)
$$

provided $X_{0}$ is close enough to $\partial \Omega$, and $t$ is chosen small enough so that $|\nabla G|<M+\delta$ on $\left\{X: G_{0}(X)=t\right\}$. Let $E(t)=\{X:|\nabla G(X)|>M-2 \delta\} \cap$ $\left\{X: G_{0}(X)=t\right\}$.

First one shows that $E(t)$ is a "large" set at "distance" comparable to $t$ from $\partial \Omega$. More precisely for $X \in E(t)$,

$$
C_{1} d(X) \leq t \leq C_{2} d(X)
$$

where $C_{i}=C\left(n, K_{0}, R, X_{0}\right)$ for $i=1,2$. Furthermore for $t$ small enough there exist balls $\left\{B\left(X_{i}, d\left(X_{i}\right)\right)\right\}$ with $X_{i}=X_{i}(t) \in E(t)$ such that

$$
\begin{gathered}
E(t) \subset \bigcup_{i} B\left(X_{i}, \frac{d\left(X_{i}\right)}{4}\right) \\
B\left(X_{i}, \frac{d\left(X_{i}\right)}{100}\right) \cap B\left(X_{j}, \frac{d\left(X_{j}\right)}{100}\right)=\emptyset \quad \text { for } i \neq j \\
\sum_{i} d\left(X_{i}\right)^{n} \geq C_{3}^{-1},
\end{gathered}
$$

where $C_{3}=C\left(X_{0}, K_{0}, n, R\right)$. Note that each $B\left(X_{i}, d\left(X_{i}\right)\right)$ is tangent to $\partial \Omega$. 
Let $\gamma>0$ be a small positive constant. Since $\Omega$ is a set of locally finite perimeter, Egoroff's theorem ensures that there exits $r_{\gamma}>0$ so that

$$
\frac{\mathcal{H}^{n}(\partial \Omega \cap B(Z, r))}{\omega_{n} r^{n}}<1+\gamma \text { for } 0<r<r_{\gamma}
$$

whenever $Z \in \partial \Omega \backslash \Lambda$ and $\mathcal{H}^{n}(\Lambda)<\gamma^{100 n}$. Choosing $t \ll r_{\gamma}$ (3.17), (3.18), (3.19) and Lemma 3 in [10] guarantee that there exists $Y \in E(t)$ so that

$$
|\nabla G(X)-\nabla G(Y)| \leq \gamma \quad \forall X \in B(Y ;(1-\gamma) d(Y))
$$

and if $\widehat{Z} \in \partial \Omega \cap \partial B(Y, d(Y))$ then there exists $Z \in \partial \Omega$ such that $|Z-\widehat{Z}|<\gamma t$ and $Z$ satisfies (3.20). For complete details see [10] Section 3.

For $0<r<r_{\gamma}(3.5),(3.8),(3.9),(3.21)$ and the fact that $t \sim d(Y)$ yield

$$
\begin{aligned}
f_{\partial B(\widehat{Z}, r)} G d \sigma & \leq f_{\partial B(Z, r)} G d \sigma+C_{n} K_{0} \gamma t \\
& \leq \frac{1}{(n+1) \omega_{n+1}} \int_{0}^{r} \frac{\omega(B(Z, s))}{s^{n}} d s+C_{n} K_{0} \gamma d(Y) \\
& \leq \frac{e^{\varepsilon}}{(n+1) \omega_{n+1}} \int_{0}^{r} \frac{\mathcal{H}^{n}(B(Z, s) \cap \partial \Omega)}{s^{n}} d s+C_{n} K_{0} \gamma d(Y) \\
& \leq \frac{e^{\varepsilon} \omega_{n}}{(n+1) \omega_{n+1}}(1+\gamma) r+C_{n} K_{0} \gamma d(Y)
\end{aligned}
$$

Assume $\widehat{Z}=Y-d(Y) e$, from (3.5) and (3.21) we deduce for $X \in B(Y, d(Y))$

$$
|G(X)-G(Y)-\langle\nabla G(Y) ; X-Y\rangle| \leq C_{n} K_{0} \gamma d(Y) .
$$

For $X=\widehat{Z}$ we have

$$
|G(Y)-\langle\nabla G(Y) ; d(Y) e\rangle| \leq C_{n} K_{0} \gamma d(Y) .
$$

Combining (3.23) and (3.24) and using the fact that $G \geq 0$ we obtain for $X \in B(Y, d(Y))$

$$
-\langle\nabla G(Y), e\rangle d(Y)-\langle\nabla G(Y) ; X-Y\rangle \leq 2 C_{n} K_{0} \gamma d(Y) .
$$

Since $Y \in E(t),|\nabla G(Y)| \neq 0$, letting $X$ tend to $Y-d(Y) \nabla G(Y) /|\nabla G(Y)|$ we obtain

$$
0 \leq|\nabla G(Y)|-\langle\nabla G(Y), e\rangle \leq 2 C_{n} K_{0} \gamma .
$$

Combining (3.23), (3.24) and (3.25) we find that

$$
|G(X)-\langle\nabla G(Y) ; e\rangle(\langle X-Y, e\rangle+d(Y))| \leq C_{n} K_{0} \gamma d(Y)
$$

for $X \in B(Y, d(Y))$. 
Let $r=\gamma^{1 / 2} d(Y)$. Note that

(3.28) $\mathcal{H}^{n}(\{X:\langle X-Y, e\rangle+d(Y) \geq 0\} \backslash B(Y, d(Y)) \cap \partial B(\widehat{Z}, r)) \leq C \gamma^{1 / 2} r^{n}$ and on this set

$$
\langle X-Y ; e\rangle+d(Y) \leq C \gamma^{1 / 2} r .
$$

From (3.27), (3.28), (3.29) and (3.5) we have

$$
\int_{\partial B(\widehat{Z}, r)} G(X) d \sigma(X)
$$

$\geq \int_{\partial B(\widehat{Z}, r) \cap\{X:\langle X-Y, e\rangle+d(Y) \geq 0\}} G(X) d \sigma(X)$

$\geq \int_{\partial B(\widehat{Z}, r) \cap\{X:\langle X-Y, e\rangle+d(Y) \geq 0\} \cap B(Y, d(Y))} G(X) d \sigma(X)$

$\geq\langle\nabla G(Y), e\rangle \int_{\partial B(\widehat{Z}, r) \cap\{X:\langle X-Y, e\rangle+d(Y) \geq 0\} \cap B(Y, d(Y))}(\langle X-Y, e\rangle+d(Y)) d \sigma(X)$

$$
\begin{gathered}
-C_{n} K_{0} \gamma r^{n} d(Y) \\
\geq\langle\nabla G(Y), e\rangle \int_{\partial B(\widehat{Z}, r) \cap\{X:\langle X-Y, e\rangle+d(Y) \geq 0\}}(\langle X-Y, e\rangle+d(Y)) d \sigma(X) \\
-C|\langle\nabla G(Y), e\rangle| \gamma^{1 / 2} r^{n+1}-C_{n} K_{0} \gamma r^{n} d(Y) \\
\geq\langle\nabla G(Y), e\rangle \int_{\partial B(\widehat{Z}, r) \cap\{X:\langle X-Y, e\rangle+d(Y) \geq 0\}}(\langle X-Y ; e\rangle+d(Y)) d \sigma(X) \\
-C_{n} K_{0} \gamma^{1 / 2} r^{n+1} .
\end{gathered}
$$

Note that

$$
\begin{aligned}
\int_{\partial B(\widehat{Z}, r) \cap\{X:\langle X-Y ; e\rangle+d(Y) \geq 0\}} & (\langle X-Y, e\rangle+d(Y)) d \sigma(X) \\
& =\int_{\partial B(0, r) \cap\left\{X: x_{n+1} \geq 0\right\}} x_{n+1} d \sigma(X) .
\end{aligned}
$$

The representation formula for subharmonic functions applied to $V(X)=$ $\max \left\{x_{n+1}, 0\right\}$ yields

$$
\int_{\partial B(0, r) \cap\left\{X: x_{n+1} \geq 0\right\}} x_{n+1} d \sigma(X)=r^{n} \int_{0}^{r} \frac{\omega_{n} s^{n}}{s^{n}} d s=\omega_{n} r^{n+1} .
$$


Combining (3.30), (3.31) and (3.32) we have

$$
\int_{\partial B(\widehat{Z}, r)} G(X) d \sigma(X) \geq\langle\nabla G(Y), e\rangle \omega_{n} r^{n+1}-C_{n} K_{0} \gamma^{1 / 2} r^{n+1} .
$$

From (3.22) and (3.33) we deduce

$$
\begin{aligned}
\langle\nabla G(Y), e\rangle \frac{\omega_{n}}{(n+1) \omega_{n+1}} r & -C_{n} K_{0} \gamma^{1 / 2} r \\
& \leq \frac{\omega_{n}}{(n+1) \omega_{n}} e^{\varepsilon}(1+\gamma) r+C_{n} K_{0} \gamma^{1 / 2} r
\end{aligned}
$$

thus

$$
\langle\nabla G(Y), e\rangle \leq e^{\varepsilon}(1+\gamma)+C_{n} K_{0} \gamma^{1 / 2}
$$

Using the fact that $Y \in E(t),(3.26)$ and (3.35) we conclude that

$$
M-2 \delta \leq|\nabla G(Y)| \leq\langle\nabla G(Y), e\rangle+2 C_{n} K_{0} \gamma \leq e^{\varepsilon}(1+\gamma)+C_{n} K_{0} \gamma^{1 / 2}
$$

Since $\gamma>0$ is arbitrary we conclude from (3.36) that $M-2 \delta \leq e^{\varepsilon}$. Letting $\delta$ tend to 0 we get that $M \leq e^{\varepsilon}$, which contradicts our initial assumption that $M>e^{\varepsilon}$. This remark finishes the proof of the main lemma.

Let

$$
\begin{aligned}
& 0<R_{1}=\sup \{r: B(0, r) \subset \Omega\}<\infty \\
& 0<R_{2}=\inf \{r: \Omega \subset B(0, r)\}<\infty .
\end{aligned}
$$

To estimate $R_{1}$, let $P_{1}=\partial \Omega \cap \partial B\left(0, R_{1}\right)$. Let $G_{1}$ be the Green's function of $B\left(0, R_{1}\right)$ with pole 0 , let $G$ be the Green's function of $\Omega$ with pole 0 . By the maximum principle for $X \in B\left(0, R_{1}\right) \backslash\{0\}$

$$
G_{1}(X) \leq G(X)
$$

In fact if $F(X)$ denotes the fundamental solution for the Laplacian in $\mathbb{R}^{n+1}$ with pole at the origin then $G=F-u$ and $G_{1}=F-u_{1}$ where $\Delta u=0$ in $\Omega$ with $u=F$ on $\partial \Omega$ and $\Delta u_{1}=0$ in $B\left(0, R_{1}\right)$ with $u_{1}=F$ on $\partial B\left(0, R_{1}\right)$. Since $G \geq 0$ then $u \leq F$ in $\Omega$, and hence $u \leq u_{1}$ on $\partial B\left(0, R_{1}\right)$ (because $B\left(0, R_{1}\right) \subset$ $\Omega)$. By the maximum principle $u \leq u_{1}$ in $B\left(0, R_{1}\right)$ which justifies (3.39). Letting $X=t P_{1}$ with $t \rightarrow 1$ (3.39) yields

$$
\liminf _{t \rightarrow 1} \frac{G_{1}\left(t P_{1}\right)}{t} \leq \liminf _{t \rightarrow 1} \frac{G\left(t P_{1}\right)}{t} .
$$


Thus by (2.10) and the Main Lemma we have that

$$
\frac{1}{\mathcal{H}^{n}\left(\partial B\left(0, R_{1}\right)\right)}=\left|\nabla G_{1}\left(P_{1}\right)\right| \leq e^{\varepsilon} .
$$

If $\sigma_{n}=\mathcal{H}^{n}(\partial B(0,1))$ then $(3.41)$ implies

$$
\left(e^{-\varepsilon} \sigma_{n}^{-1}\right)^{1 / n} \leq R_{1} .
$$

To estimate $R_{2}$ let $P_{2} \in \partial \Omega$ be such that $\left|P_{2}\right|=\max \{|Q|: Q \in \partial \Omega\}$. Let $G_{2}$ denote the Green's function of $B\left(0, R_{2}\right)$ with pole at 0 . A similar argument to the one above shows that for $X \in \Omega \backslash\{0\}$

$$
G(X) \leq G_{2}(X)
$$

Note that for $P_{2}$ there exists a ball $B \subset \Omega^{c}$ such that $P_{2} \in \partial \Omega \cap \partial B$.

Lemma 3.2 Let $\Omega, G$ and $h$ be as above. Let $P \in \partial \Omega$ and assume that there exists a ball $B \subset \Omega^{c}=\{G=0\}$ so that $P \in \partial \Omega \cap \partial B$ then

$$
\limsup _{\substack{X \rightarrow P \\ X \in \Omega}} \frac{G(X)}{d(X, B)} \geq e^{-\varepsilon}
$$

Proof. Let

$$
l=\limsup _{\substack{X \rightarrow P \\ X \in \Omega}} \frac{G(X)}{d(X, B)} .
$$

There exists a sequence $\left\{Y_{k}\right\}_{k \geq 1} \subset \Omega$, such that $Y_{k} \rightarrow P$ and $\frac{G\left(Y_{k}\right)}{d\left(Y_{k}, B\right)} \rightarrow l$ as $k \rightarrow \infty$. Let $d_{k}=d\left(Y_{k}, B\right)$. There exists $X_{k} \in \partial B$ so that $\left|Y_{k}-X_{k}\right|=d_{k}$. Consider

$$
G_{k}(X)=\frac{G\left(d_{k} X+X_{k}\right)}{d_{k}} \quad \text { for } X \in B(0,2) \quad \text { and } \quad Z_{k}=\frac{Y_{k}-X_{k}}{d_{k}} .
$$

Without loss of generality we may assume that $Z_{k} \rightarrow e$ as $k \rightarrow \infty,|e|=1$ and $G_{k} \underset{k \rightarrow \infty}{\longrightarrow} G_{\infty}$ in $C_{\text {loc }}^{0, \beta}\left(\mathbb{R}^{n+1}\right), \nabla G_{k} \underset{k \rightarrow \infty}{\stackrel{*}{\rightarrow}} \nabla G_{\infty}$ weak star in $L_{\text {loc }}^{\infty}\left(\mathbb{R}^{n+1}\right)$, weakly in $L_{\text {loc }}^{2}\left(\mathbb{R}^{n+1}\right)$;

$$
\frac{1}{d_{k}}\left(\partial \Omega-X_{k}\right)=\partial\left\{G_{k}>0\right\} \underset{k \rightarrow \infty}{\longrightarrow} \partial\left\{G_{\infty}>0\right\}
$$

in the Hausdorff distance sense uniformly on compact sets, and $\chi_{\left\{G_{k}>0\right\}} \rightarrow$ $\chi_{\left\{G_{\infty}>0\right\}}$ in $L_{\text {loc }}^{1}\left(\mathbb{R}^{n+1}\right)$. Note that $G_{k}\left(Z_{k}\right)=\frac{G\left(Y_{k}\right)}{d_{k}}$ thus $G_{k}\left(Z_{k}\right) \rightarrow l$ as $k \rightarrow \infty$. On the other hand since $G_{k}$ converges uniformly to $G_{\infty}$ in $B(0,2)$, we conclude that $G_{\infty}(e)=l$. 
In order to prove the lemma we need to get a better understanding of $G_{\infty}$ and $\Omega_{\infty}=\left\{G_{\infty}>0\right\}$. Our goal is to show that $\Omega_{\infty}$ is a half-space and $G_{\infty}$ is linear. Let $r$ be the radius of $B$. Let $\alpha_{k}=d\left(\partial B\left(X_{k}, d_{k}\right) \cap \partial B ; L\right)$, where $L$ is the tangent plane to $B$ through $X_{k}$. An easy computation shows that $\alpha_{k}=2 \frac{d_{k}^{2}}{r}$. Note that for $P_{k} \in B\left(X_{k}, d_{k}\right) \cap\left\{\left\langle P-X_{k}, \frac{Y_{k}-X_{k}}{d_{k}}\right\rangle<-\alpha_{k}\right\} \subset B$ if $Q_{k}=\frac{P_{k}-X_{k}}{d_{k}}$, then $Q_{k} \in B(0,2) \cap\left\{\left\langle X, Z_{k}\right\rangle<-\frac{d_{k}}{r}\right\}$ and $G_{k}\left(Q_{k}\right) \leq 0$. Passing to the limit as $k$ tends to infinity we conclude that if $Y \in B(0,2) \cap$ $\{\langle Y, e\rangle \leq 0\}$ then $G_{\infty}(Y)=0$. Let $Y \in B(0,2) \cap\left\{\left\langle Y, Z_{k}\right\rangle>0\right\}$, then either $d_{k} Y+X_{k} \in \Omega^{c}$ and $G_{k}(Y)=0$ or $d_{k} Y+X_{k} \in \Omega$ and given $\varepsilon>0$ there exists $k_{0} \in \mathbb{N}$ such that for $k \geq k_{0}$

$$
\frac{G\left(d_{k} Y+X_{k}\right)}{d\left(d_{k} Y+X_{k}, B\right)} \leq l+\varepsilon
$$

and

$$
\begin{aligned}
G\left(d_{k} Y+X_{k}\right) & \leq(l+\varepsilon) d\left(d_{k} Y+X_{k}, B\right) \\
& \leq(l+\varepsilon)\left\{\left\langle d_{k} Y ; \frac{Y_{k}-X_{k}}{d_{k}}\right\rangle+2 \frac{d_{k}^{2}}{r}\right\} \\
& \leq(l+\varepsilon) d_{k}\left\{\left\langle Y, Z_{k}\right\rangle+2 \frac{d_{k}}{r}\right\}
\end{aligned}
$$

which implies

$$
G_{k}(Y)=\frac{G\left(d_{k} Y+X_{k}\right)}{d_{k}} \leq(l+\varepsilon)\left\{\left\langle Y, Z_{k}\right\rangle+2 \frac{d_{k}}{r}\right\} .
$$

Passing to the limit as $k$ goes to infinity we conclude that for $Y \in B(0,2) \cap$ $\{\langle Y, e\rangle \geq 0\}, G_{\infty}(Y) \leq(l+\varepsilon)\langle Y, e\rangle$ for every $\varepsilon>0$, thus $G_{\infty}(Y) \leq l\langle Y, e\rangle$. Moreover $G_{\infty}(e)=l$. The maximum principle guarantees that $v_{\infty}(Y)=$ $l \max \{\langle Y, e\rangle ; 0\}$ for $Y \in B(0,1)$.

If $h_{k}(X)=h\left(d_{k} X+X_{k}\right)$, for $\zeta \in C_{c}^{\infty}(B(1,0)), \zeta \geq 0$

$$
\begin{aligned}
\int_{\partial\left\{G_{k}>0\right\}} \zeta h_{k} d \mathcal{H}^{n} & =\int_{\mathbb{R}^{n+1}} \nabla G_{k} \cdot \nabla \zeta \\
& \longrightarrow-\int_{k \rightarrow \infty} \nabla G_{\infty} \cdot \nabla \zeta=\int_{\{\langle Y, e\rangle=0\}} l \zeta d \mathcal{H}^{n}
\end{aligned}
$$

thus

$$
\lim _{k \rightarrow \infty} \int_{\partial\left\{G_{k}>0\right\}} \zeta h_{k} d \mathcal{H}^{n}=l \int_{\{\langle Y, e\rangle=0\}} \zeta d H^{n} .
$$


On the other hand the divergence theorem ensures that

$$
\int_{\partial\left\{G_{k}>0\right\}} \zeta d \mathcal{H}^{n} \geq \int_{\partial\left\{G_{k}>0\right\}} \zeta e \cdot \nu_{k} d \mathcal{H}^{n}=\int_{\left\{G_{k}>0\right\}} \operatorname{div}(\zeta e) .
$$

Since

(3.51) $\int_{\left\{G_{k}>0\right\}} \operatorname{div}(\zeta e) \underset{k \rightarrow \infty}{\longrightarrow} \int_{\left\{G_{\infty}>0\right\}} \operatorname{div}(\zeta e)=\int_{\partial\left\{G_{\infty}>0\right\}} \zeta d \mathcal{H}^{n}=\int_{\langle Y, e\rangle=0} \zeta d \mathcal{H}^{n}$,

we have that

$$
\lim _{k \rightarrow \infty} \int_{\partial\left\{G_{k}>0\right\}} \zeta d \mathcal{H}^{n} \geq \int_{\{\langle Y, e\rangle=0\}} \zeta d \mathcal{H}^{n} .
$$

Since by (3.2), $h \geq e^{-\varepsilon} \mathcal{H}^{n}-$ a.e. $Q \in \partial \Omega$, using (3.49) and (3.52) we have

$$
\begin{aligned}
\lim _{k \rightarrow \infty} \int_{\partial\left\{G_{k}>0\right\}} h_{k} \zeta d \mathcal{H}^{n} & \geq \lim _{k \rightarrow \infty} \int_{\partial\left\{G_{k}>0\right\}} e^{-\varepsilon} \zeta d \mathcal{H}^{n} \\
l \int_{\{\langle Y, e\rangle=0\}} \zeta d \mathcal{H}^{n} & \geq e^{-\varepsilon} \int_{\{\langle Y, e\rangle=0\}} \zeta d \mathcal{H}^{n},
\end{aligned}
$$

for any $\zeta \in C_{c}^{\infty}(B(1,0)), \zeta \geq 0$. Therefore (3.53) yields

$$
l \geq e^{-\varepsilon} \text {. }
$$

Combining (3.43) and (3.44) we obtain that

$$
\left|\nabla G_{2}\left(P_{2}\right)\right| \geq \limsup _{\substack{X \rightarrow P_{2} \\ X \in \Omega}} \frac{G(X)}{d(X, B)} \geq e^{-\varepsilon} .
$$

Thus

$$
\frac{1}{\mathcal{H}^{n}\left(\partial B\left(0, R_{2}\right)\right)}=\frac{1}{\sigma_{n} R_{2}^{n}} \geq e^{-\varepsilon}
$$

which implies

$$
R_{2} \leq\left(e^{\varepsilon} \sigma_{n}^{-1}\right)^{\frac{1}{n}}
$$

We have proved the following lemma.

Lemma 3.3 Assume that $\Omega \subset \mathbb{R}^{n+1}$ satisfies conditions (2.1) and (2.2) in Theorem 2.1 then

$$
B\left(0, R_{1}\right) \subset \Omega \subset B\left(0, R_{2}\right)
$$

with

$$
e^{-\varepsilon} \leq \sigma_{n} R_{1}^{n} \leq \sigma_{n} R_{2}^{n} \leq e^{\varepsilon} .
$$




\section{Fine Geometric Properties}

In this section we prove Theorem 2.1. For this purpose we first introduce a local notion of flatness that involves the geometry of the boundary at a point $Q_{0}$, the behavior of $G$ near $Q_{0}$ and the oscillation of $\log h$ near this point (see Definition 7.1 in [1]). We assume that $G$ is continuously extended to be identically 0 outside $\Omega$. Note that $G$ is then subharmonic in $\mathbb{R}^{n+1}$.

Definition 4.1 Let $\Omega \subset \mathbb{R}^{n+1}$ be as in Theorem 2.1. Let $Q_{0} \in \partial \Omega$, $\rho>0$ and $\sigma_{+}, \sigma_{-}, \tau \in(0,1)$. We say that

$$
G(X) \geq-h\left(Q_{0}\right)\left[\left\langle X-Q_{0}, \nu\right\rangle+\sigma_{-} \rho\right] \text { for }\left\langle X-Q_{0} ; \nu\right\rangle \leq-\sigma_{-} \rho
$$

and

$$
\sup _{X \in B\left(Q_{0}, \rho\right)}|\nabla G(X)| \leq h\left(Q_{0}\right)(1+\tau) \text { and } \underset{B\left(Q_{0}, \rho\right)}{\operatorname{Osc} h} \leq \tau h\left(Q_{0}\right) .
$$

The proof is very similar to the ones presented in [1] section 7 or in [6]. To avoid repetition we state the lemmata and only point out the main differences with respect to the proofs of the results mentioned above. For the complete details we refer the reader to [1] and [6].

Lemma 4.1 Let $\Omega \subset \mathbb{R}^{n+1}$ be a bounded domain and a set of locally finite perimeter such that $0 \in \Omega$. Let $G$ and $h$ be as above. There exists $\sigma_{n}>0$ so that if $\sigma \in\left(0, \sigma_{n}\right), \tau \in(0, \sigma)$ and $\varepsilon \in(0, \sigma)$ with

$$
\sup _{\partial \Omega}|\log h|<\varepsilon
$$

then for $Q_{0} \in \partial \Omega, \rho>0$ and $\nu \in \mathbb{S}^{n}$, if $G \in F(\sigma, 1 ; \tau)$ in $B\left(Q_{0}, \rho\right)$ in direction $\nu$ then $G \in F(2 \sigma, C \sigma ; \tau)$ in $B\left(Q_{0}, \frac{\rho}{2}\right)$ in direction $\nu$. Here $C>1$ is a constant that only depends on $n$.

Lemma 4.2 Let $\Omega \subset \mathbb{R}^{n+1}$ be a bounded domain and a set of locally finite perimeter such that $0 \in \Omega$. Let $G$ and $h$ be as above. Given $\theta \in(0,1)$ there exists $\sigma_{\theta}>0$ and $\eta_{\theta}=\eta \in(0,1)$ so that if $\sigma \in\left(0, \sigma_{\theta}\right)$ and $\tau \in\left(0, \sigma_{\theta} \sigma^{2}\right)$ then for $Q_{0} \in \partial \Omega, \rho>0$ if $G \in F(\sigma, \sigma ; \tau)$ in $B\left(Q_{0}, \rho\right)$ in direction $\nu$ then $G \in F(\theta \sigma, 1 ; \tau)$ in $B\left(Q_{0}, \eta \rho\right)$ in direction $\bar{\nu}$ and $|\nu-\bar{\nu}| \leq C \sigma$. 
Lemma 4.3 Assume that $\Omega \subset \mathbb{R}^{n+1}$ satisfies (2.9). Then given $\sigma>0$ there exist $\varepsilon_{\sigma}>0$ such that if

$$
\sup _{\partial \Omega}|\log h|<\varepsilon \text { with } \varepsilon<\varepsilon_{\sigma}
$$

then there is $\rho_{\varepsilon}=\rho>0$ (depending on $\varepsilon>0$ ) so that for $Q \in \partial \Omega, G \in$ $F\left(\sigma, \sigma ;\left(e^{2 \varepsilon}-1\right)^{1 / 4}\right)$ in $B(Q, \rho)$. Here $\left(e^{2 \varepsilon_{\sigma}}-1\right)^{1 / 2}<\sigma$.

Proof of Lemma 4.3. Recall from Lemma 3.3 that under the above hypothesis $B\left(0, R_{1}\right) \subset \Omega \subset B\left(0, R_{2}\right)$ with $1 \leq R_{2} / R_{1} \leq e^{2 \varepsilon}$, and $e^{-\varepsilon} \leq \sigma_{n} R_{i}^{n} \leq$ $e^{\varepsilon}$ for $i=1,2$. Let $\varepsilon \in\left(0, \frac{1}{4}\right)$ be a positive number to be chosen later depending on $\sigma>0$. Let $\rho=R_{1} \sqrt{2} \sqrt{e^{2 \varepsilon}-1}$. From basic geometry and the remark above (see Lemma 3.3) it is clear that for $Q \in \partial \Omega$ there exists an $n$-plane $L(Q, \rho)$ through $Q$ such that

$$
\frac{1}{\rho} D[\partial \Omega \cap B(Q, \rho), L(Q, \rho) \cap B(Q, \rho)] \leq \sqrt{2} \sqrt{e^{2 \varepsilon}-1} .
$$

In fact take for example the $n$-plane through $Q$ orthogonal to the line joining the origin to $Q$. Let $\nu$ be the unit normal in the direction $\overrightarrow{O Q}$ we have that if $X \in B(Q, \rho)$ and $\langle X-Q, \nu\rangle \geq 2 \sqrt{2} \sqrt{e^{2 \varepsilon}-1} \rho$ then since $1 \leq R_{2} / R_{1} \leq e^{2 \varepsilon}$

$$
\begin{aligned}
|X|^{2} & =|X-Q-\langle X-Q, \nu\rangle \nu|^{2}+|\langle X-Q, \nu\rangle+| Q||^{2} \\
& \geq\left(R_{1}+2 \sqrt{2} \sqrt{e^{2 \varepsilon}-1} \rho\right)^{2}=R_{1}^{2}\left(1+4\left(e^{2 \varepsilon}-1\right)\right)^{2} \\
& \geq R_{2}^{2} e^{-4 \varepsilon}\left(4 e^{2 \varepsilon}-3\right)^{2} \\
& \geq R_{2}^{2}\left(4-3 e^{-2 \varepsilon}\right)^{2}>R_{2}^{2} .
\end{aligned}
$$

Thus $X \notin \Omega$ and $G(X)=0$ as $G$ was extended to be identically equal to zero in $\Omega^{c}$. Now let $X \in B(Q, \rho)$ with $\langle X-Q, \nu\rangle \leq-2 \sqrt{2} \sqrt{e^{2 \varepsilon}-1} \rho$. In this case

$$
\begin{aligned}
|X|^{2} & =|X-Q-\langle X-Q, \nu\rangle \nu|^{2}+|\langle X-Q, \nu\rangle+| Q||^{2} \\
& \leq|X-Q|^{2}+\left(R_{2}-4\left(e^{2 \varepsilon}-1\right) R_{1}\right)^{2} \\
& \leq \rho^{2}+\left(e^{2 \varepsilon}-4 e^{2 \varepsilon}+4\right)^{2} R_{1}^{2} \\
& \leq\left[2\left(e^{2 \varepsilon}-1\right)+\left(1-3\left(e^{2 \varepsilon}-1\right)\right)^{2}\right] R_{1}^{2} \\
& \leq\left(1+9\left(e^{2 \varepsilon}-1\right)^{2}-4\left(e^{2 \varepsilon}-1\right)\right) R_{1}^{2} \\
& \leq\left[1+\left(e^{2 \varepsilon}-1\right)\left(9\left(e^{2 \varepsilon}-1\right)-4\right)\right] R_{1}^{2}<R_{1}^{2}
\end{aligned}
$$

provided $\varepsilon>0$ is such that $e^{2 \varepsilon}-1<4 / 9$. Thus for $X \in B(Q, \rho)$ with $\langle X-Q, \nu\rangle \leq-2 \sqrt{2} \sqrt{e^{2 \varepsilon}-1} \rho, X \in B\left(0, R_{1}\right)$ and by (3.39) we have that if $G_{1}$ 
denotes the Green function of $B\left(0, R_{1}\right)$ with pole 0 then

$$
\begin{aligned}
G(X) & \geq G_{1}(X)=G_{1}(X)-G_{1}\left(R_{1} \frac{X}{|X|}\right) \\
& \geq\left\langle\nabla G_{1}(Y), X-R_{1} \frac{X}{|X|}\right\rangle=\left\langle\nabla G_{1}(Y), \frac{X}{|X|}\right\rangle\left(|X|-R_{1}\right),
\end{aligned}
$$

for some $Y=t X+(1-t) R_{1} \frac{X}{|X|}$ with $t \in[0,1]$. Note that $R_{1}-\rho \leq|Y| \leq R_{1}$. The last inequality is a simple application of the fundamental theorem of calculus.

Since $G_{1}(Y)=\frac{1}{(n-1) \sigma_{n}}\left(\frac{1}{|Y|^{n-1}}-\frac{1}{R_{1}^{n-1}}\right), \nabla G_{1}(Y)=\frac{-1}{\sigma_{n}} \frac{Y}{|Y|^{n+1}}$ and for $Y$ as above (4.10) and (3.59) ensure

$$
\begin{aligned}
G(X) & \geq \frac{-1}{\sigma_{n}|X|^{n}}\left(t+(1-t) \frac{R_{1}}{|X|}\right)^{-n}\left(|X|-R_{1}\right) \\
& \geq \frac{1}{\sigma_{n}|Y|^{n}}\left(R_{1}-|X|\right) \geq \frac{1}{\sigma_{n} R_{1}^{n}}\left(R_{1}-|X|\right) \\
& \geq e^{-\varepsilon}\left(R_{1}-|X|\right)
\end{aligned}
$$

Combining (4.10), (4.11) and (4.6) we obtain

$$
\begin{aligned}
G(X) & \geq e^{-\varepsilon}\left(R_{1}-|X|\right) \\
& \geq h(Q)\left(R_{1}-|X|\right)-h(Q)\left(1-e^{-2 \varepsilon}\right)\left(R_{1}-|X|\right) \\
& \geq h(Q)\left(R_{1}-|X|\right)-h(Q)\left(1-e^{-2 \varepsilon}\right) \rho,
\end{aligned}
$$

for $X \in B(Q, \rho)$ with $\langle X-Q, \nu\rangle \leq-2 \sqrt{2} \sqrt{e^{2 \varepsilon}-1} \rho$.

Our next goal is to compare $R_{1}-|X|$ to $|\langle X-Q, \nu\rangle|$. Note that the basic picture is as follows:

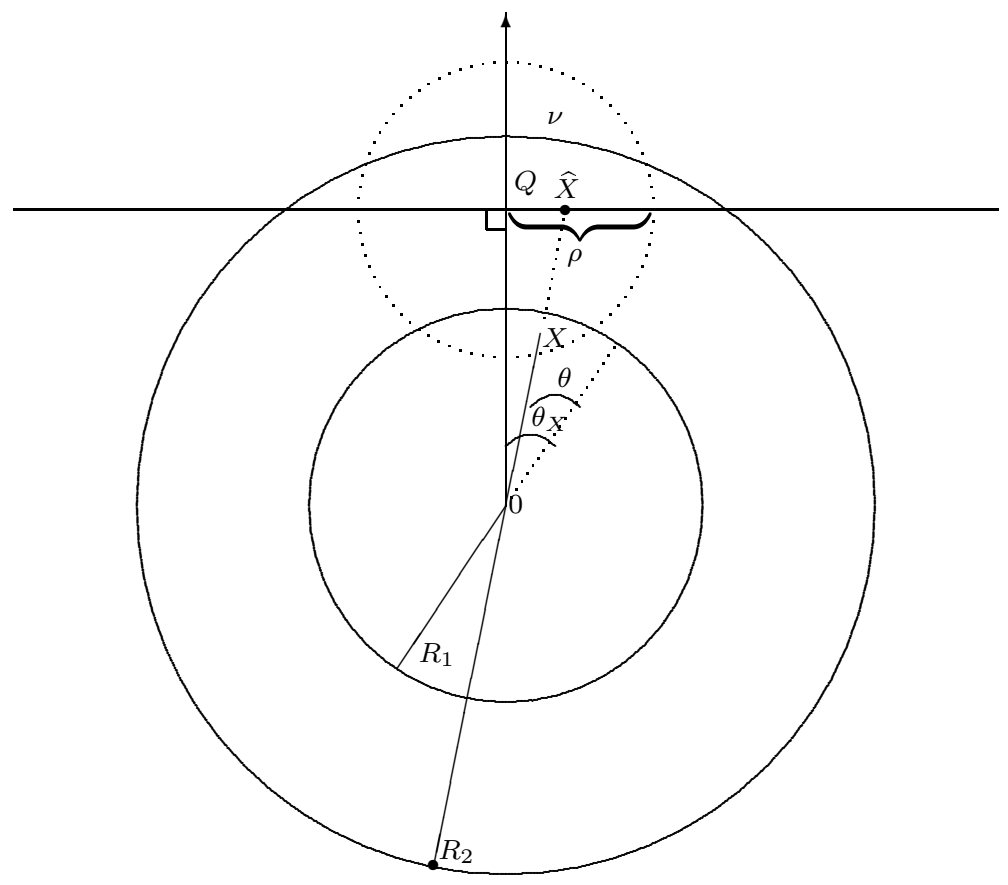


where

$$
|\langle X-Q, \nu\rangle|=\cos \theta_{X}|X-\widehat{X}| \leq\left(R_{1}-|X|+\frac{R_{2}-R_{1}}{\cos \theta_{X}}\right) \cos \theta_{X} .
$$

Thus since $\langle X-Q, \nu\rangle \leq-2 \sqrt{2} \sqrt{e^{2_{\varepsilon}}-1} \rho \leq 0$ and $R_{1} \leq R_{2} \leq e^{2 \varepsilon} R_{1}$

$$
\begin{aligned}
R_{1}-|X| & \geq \frac{1}{\cos \theta_{X}}|\langle X-Q, \nu\rangle|-\left(R_{2}-R_{1}\right) \\
& \geq \frac{1}{\cos \theta_{X}}|\langle X-Q, \nu\rangle|-R_{1}\left(e^{2 \varepsilon}-1\right) \\
& \geq \frac{1}{\cos \theta_{X}}|\langle X-Q, \nu\rangle|-\frac{\sqrt{e^{2 \varepsilon}-1}}{\sqrt{2}} \rho \\
& \geq|\langle X-Q, \nu\rangle|-\frac{e^{2 \varepsilon}-1}{\sqrt{2} \rho .}
\end{aligned}
$$

Combining (4.6), (4.12) and (4.14) we have that for $X \in B(Q, \rho)$ with $\langle X-Q ; \nu\rangle \leq-2 \sqrt{2} \sqrt{e^{2 \varepsilon}-1} \rho$

$$
\begin{aligned}
G(X) & \geq h(Q)\left(R_{1}-|X|\right)-h(Q)\left(1-e^{-2 \varepsilon}\right) \rho \\
& \geq h(Q)\left[|\langle X-Q, \nu\rangle|-\frac{e^{2 \varepsilon}-1}{\sqrt{2}} \rho-h(Q)\left(1-e^{-2 \varepsilon}\right)\right] \\
& \geq h(Q)\left[-\langle X-Q, \nu\rangle-\frac{1+\sqrt{2}}{\sqrt{2}}\left(e^{2 \varepsilon}-1\right) \rho\right] .
\end{aligned}
$$

Thus choosing $\varepsilon>0$ so that $2 \sqrt{2} \sqrt{e^{2 \varepsilon}-1}<\left(e^{2 \varepsilon}-1\right)^{\frac{1}{12}}<\sigma$ and we have that for $X \in B(Q, \rho)$

$$
\begin{gathered}
G(X)=0 \text { for }\langle X-Q, \nu\rangle \geq \sigma \rho \\
G(X) \geq-h(X)[\langle X-Q, \nu\rangle+\sigma \rho] \text { for }\langle X-Q, \nu\rangle \leq-\sigma \rho .
\end{gathered}
$$$$
\text { Hypothesis (4.6) implies that for } P, Q \in \partial \Omega
$$

$$
e^{-2 \varepsilon} \leq \frac{h(P)}{h(Q)} \leq e^{2 \varepsilon}
$$

Thus

$$
\operatorname{OSc}_{B(Q, P)} h \leq\left(e^{2 \varepsilon}-1\right) h(Q) .
$$

To estimate $\sup _{B(Q, P) \cap \Omega}|\nabla G|$ recall that the function $V(X)=|\nabla G(X)|$ is subharmonic and bounded on $\Omega \backslash B\left(0, \frac{R_{1}}{2}\right)$. Hence, since

$$
B(Q, \rho) \cap \Omega \subset \Omega \backslash B\left(0, R_{1}-2 \rho\right),
$$


the maximum principle for subharmonic functions ensures that

$$
\begin{aligned}
\sup _{B(Q, \rho) \cap \Omega}|\nabla G| & \leq \sup _{\Omega \backslash B\left(Q, R_{1}-2 \rho\right)}|\nabla G| \\
& =\max \left\{\limsup _{X \rightarrow \partial \Omega}|\nabla G(X)|, \sup _{\partial B\left(0, R_{1}-2 \rho\right)}|\nabla G|\right\} .
\end{aligned}
$$

Let $Y \in \partial B\left(0, R_{1}-2 \rho\right)$ then $B(Y, \rho) \subset B\left(0, R_{1}\right) \subset \Omega$ since $G$ and $G_{1}$ are harmonic on $B(Y, \rho)$ Poisson's representation formula yields for $X \in B(Y, \rho)$

$$
G(X)=\frac{\rho^{2}-|X-Y|^{2}}{(n+1) \omega_{n+1} \rho} \int_{\partial B(Y, \rho)} \frac{G(\zeta)}{|X-\zeta|^{n+1}} d \zeta
$$

Differentiating the expression in (4.21) and applying the obtained formula to $X=Y$ we obtain

$$
\begin{aligned}
\nabla G(Y) & =-\frac{\rho}{\omega_{n+1}} \int_{\partial B(Y, \rho)} \frac{G(\zeta)}{|Y-\zeta|^{n+3}}(Y-\zeta) d \zeta \\
& =-\frac{1}{\omega_{n+1} \rho^{n+2}} \int_{\partial B(Y, \rho)} G(\zeta)(Y-\zeta) d \zeta .
\end{aligned}
$$

Thus if $G_{i}$ denotes the Green function of $B\left(0, R_{i}\right)$ for $i=1,2$ with pole 0 , we have

$$
\left|\nabla G(Y)-\nabla G_{1}(Y)\right| \leq \frac{\rho}{\omega_{n+1} \rho^{n+2}} \int_{\partial B(Y, \rho)}\left|G(\zeta)-G_{1}(\zeta)\right| d \zeta .
$$

Using (3.39), (3.43) and (4.23) we have

$$
\begin{aligned}
\mid \nabla G(Y)- & \nabla G_{1}(Y) \mid \leq \frac{1}{\omega_{n+1} \rho^{n+1}} \int_{\partial B(Y, \rho)}\left(G_{2}(\zeta)-G_{1}(\zeta)\right) d \zeta \\
& \leq C_{n} \frac{1}{\rho^{n+1}} \int_{\partial B(Y, \rho)}\left(\frac{1}{R_{1}^{n-1}}-\frac{1}{R_{2}^{n-1}}\right) d \zeta \\
& \leq \frac{C_{n}}{\rho}\left[\frac{1}{R_{1}^{n-1}}-\frac{1}{R_{2}^{n-1}}\right]=\frac{C_{n}}{\rho R_{1}^{n-1} R_{2}^{n-1}}\left(R_{2}^{n-1}-R_{1}^{n-1}\right) \\
& \leq \frac{C_{n} R_{2}^{n-2}}{\rho R_{1}^{n-1} R_{2}^{n-1}}\left(R_{2}-R_{1}\right)=\frac{C_{n}}{\rho R_{1}^{n-1} R_{2}}\left(R_{2}-R_{1}\right) \\
& \leq \frac{C_{n} R_{1}\left(e^{2 \varepsilon}-1\right)}{\rho R_{1}^{n}} \leq \frac{C_{n}}{\rho} \frac{1}{R_{1}^{n-1}}\left(e^{2 \varepsilon}-1\right) \\
& \leq \frac{C_{n}}{R_{1}^{n}} \sqrt{e^{2 \varepsilon}-1} \leq C_{n} \sqrt{e^{2 \varepsilon}-1}
\end{aligned}
$$

where we used the facts that $1 \leq \frac{R_{2}}{R_{1}} \leq e^{2 \varepsilon}, \rho=\sqrt{2} \sqrt{e^{2 \varepsilon}-1} R_{1}$ and $e^{-\varepsilon} \leq$ $R_{1}^{n} \sigma_{n} \leq e^{\varepsilon}$, with $\varepsilon \in\left(0, \frac{1}{4}\right)$. 
Since

$$
G_{1}(Y)=\frac{1}{(n-1)(n+1) \omega_{n+1}}\left(\frac{1}{|Y|^{n-1}}-\frac{1}{R_{1}^{n-1}}\right)
$$

then

$$
\left|\nabla G_{1}(Y)\right|=\frac{1}{(n+1) \omega_{n+1}} \frac{1}{|Y|^{n}} .
$$

For $Y \in \partial B\left(0, R_{1}-2 \rho\right)$ and $\varepsilon>0$ small enough, we have

$$
\begin{aligned}
\left|\nabla G_{1}(Y)\right| & =\frac{1}{\omega_{n+1}(n+1)\left(R_{1}-2 \rho\right)^{n}} \\
& =\frac{1}{\sigma_{n} R_{1}^{n}\left(1-2 \sqrt{2} \sqrt{e^{2 \varepsilon}-1}\right)^{n}} \\
& \leq \frac{e^{\varepsilon}}{\left(1-2 \sqrt{2} \sqrt{e^{2 \varepsilon}-1}\right)^{n}} \\
& \leq e^{\varepsilon}\left(1+8 n \sqrt{e^{2 \varepsilon}-1}\right) .
\end{aligned}
$$

Combining (4.6), (4.20), (4.24) and (4.25) we obtain

$$
\begin{aligned}
\sup _{B(Q, \rho)}|\nabla G| & \leq e^{\varepsilon}\left(1+8 n \sqrt{e^{2 \varepsilon}-1}\right)+C_{n} \sqrt{e^{2 \varepsilon}-1} \\
& \leq e^{\varepsilon}\left(1+C_{n} \sqrt{e^{2 \varepsilon}-1}\right) \\
& \leq e^{2 \varepsilon} h(Q)\left(1+C_{n} \sqrt{e^{2 \varepsilon}-1}\right) \\
& \leq h(Q)\left(1+C_{n} \sqrt{e^{2 \varepsilon}-1}\right) .
\end{aligned}
$$

Thus for $\varepsilon>0$ small enough so that $C_{n}\left(e^{2 \varepsilon}-1\right)^{\frac{1}{4}}<1$ we have that

$$
\sup _{B(Q, \rho)}|\nabla G| \leq h(Q)\left(1+\left(e^{2 \varepsilon}-1\right)^{1 / 4}\right) .
$$

Note that (4.16), (4.17), (4.19) and (4.27) show that for $\varepsilon>0$ small enough in terms of $n$ and such that $\left(\varepsilon^{2 \varepsilon}-1\right)^{1 / 12}<\sigma$ then $G \in F\left(\sigma, \sigma ;\left(e^{2 \varepsilon}-1\right)^{1 / 4}\right)$ in $B(Q, \rho), \forall Q \in \partial \Omega$ where $\rho=\sqrt{2} \sqrt{e^{2 \varepsilon}-1} R_{1}$.

Before sketching the proofs of Lemma 4.1 and Lemma 4.2 we indicate how from the 3 lemmata above one proves Theorem 2.1 .

Proof of Theorem 2.1. Let $\theta^{\prime} \in\left(0, \frac{1}{2}\right)$ to be chosen. Let $\sigma^{\prime} \in\left(0, \sigma_{\theta^{\prime}}\right)$ as in Lemma 4.2. By Lemma 4.3 for $\sigma \in\left(0, \sigma_{\theta^{\prime}}\right)$ there is $\varepsilon_{\sigma^{\prime}}>0$ so that if (4.6) holds, then $G \in F\left(\sigma^{\prime}, \sigma^{\prime},\left(e^{2 \varepsilon}-1\right)^{1 / 4}\right)$ in $B(Q, \rho)$, for $Q \in \partial \Omega$ with $\rho=\sqrt{2} \sqrt{e^{2 \varepsilon}-1} R_{1}$, and with $\left(e^{2 \varepsilon_{\sigma^{\prime}}}-1\right)^{1 / 12}<\sigma^{\prime}$. Note that by choosing $\varepsilon^{\prime}<\varepsilon_{\sigma^{\prime}}$ so that $\left(e^{2 \varepsilon_{\sigma}}-1\right)^{1 / 4}<\sigma_{\theta^{\prime}}$ we have that $\left(e^{2 \varepsilon}-1\right)^{1 / 4} \leq \sigma_{\theta^{\prime}}\left(\sigma^{\prime}\right)^{2}$ for $\varepsilon<\varepsilon^{\prime}$. Lemma 4.2 ensures that $G \in F\left(\theta^{\prime} \sigma^{\prime}, 1 ;\left(e^{2 \varepsilon}-1\right)^{1 / 4}\right)$ in $B(Q, \eta \rho)$. 
Lemma 4.1 now guarantees that $G \in F\left(2 \theta^{\prime} \sigma^{\prime}, C \theta^{\prime} \sigma^{\prime} ;\left(e^{2 \varepsilon}-1\right)^{1 / 4}\right)$ in $B\left(Q, \frac{\eta \rho}{2}\right)$. Choosing $\theta^{\prime}$ so that $C \theta^{\prime}+2 \theta^{\prime}<1$ we conclude that

$$
G \in F\left(\sigma^{\prime}, \sigma^{\prime} ;\left(e^{2 \varepsilon}-1\right)^{1 / 4}\right) \quad \text { in } B\left(Q, \frac{\eta \rho}{2}\right) .
$$

Since $\left(e^{2 \varepsilon}-1\right)^{1 / 4} \leq \sigma_{\theta^{\prime}}\left(\sigma^{\prime}\right)^{2}$ we can repeat the previous argument to show that $\forall k \in \mathbb{N}$ and $\forall Q \in \partial \Omega$

$$
G \in F\left(\sigma^{\prime}, \sigma^{\prime} ;\left(e^{2 \varepsilon}-1\right)^{1 / 4}\right) \quad \text { in } B\left(Q,\left(\frac{\eta}{2}\right)^{k} \rho\right) .
$$

Thus there exists $\nu_{k} \in S^{n}$ so that

$$
G(X)=0 \text { for }\left\langle X-Q, \nu_{k}\right\rangle \geq \sigma^{\prime}\left(\frac{\eta}{2}\right)^{k} \rho
$$

and

$$
G(X) \geq-h(Q)\left[\left\langle X-Q, \nu_{k}\right\rangle+\sigma^{\prime}\left(\frac{\eta}{2}\right)^{k} \rho\right] \geq 0
$$

for $\left\langle X-Q, \nu_{k}\right\rangle \leq-\sigma^{\prime}\left(\frac{\eta}{2}\right)^{k} \rho$.

In particular if $L_{k}(Q)$ denotes the $n$-plane through $Q$ orthogonal to $\nu_{k}$ (4.28) and (4.29) imply that

$$
D\left[\partial \Omega \cap B\left(Q,\left(\frac{\eta}{2}\right)^{k} \rho\right) ; L_{k}(Q) \cap B\left(Q,\left(\frac{\eta}{2}\right)^{k} \rho\right)\right] \leq \sigma^{\prime}\left(\frac{\eta}{2}\right)^{k} \rho .
$$

Let $r \in(0, \rho)$ there is $k \geq 0$ so that $\left(\frac{\eta}{2}\right)^{k+1} \rho \leq r \leq\left(\frac{\eta}{2}\right)^{k} \rho$, let $r_{k}=\left(\frac{\eta}{2}\right)^{k} \rho$. For $P \in \partial \Omega \cap B(Q, r)$ by (4.30), there exists $Z \in L_{k}(Q) \cap B\left(Q, r_{k}\right)$ so that $|Z-P|<\sigma^{\prime} r_{k}$. Note that $|Z-Q| \leq|Z-P|+|P-Q|<\sigma^{\prime} r_{k}+r$. There exists $Z^{\prime} \in \operatorname{seg}[Q, Z]$ such that $\left|Z^{\prime}-Q\right|<r$ and $\left|Z^{\prime}-Z\right|<\sigma^{\prime} r_{k}$. Moreover $\left|Z^{\prime}-P\right| \leq\left|Z-Z^{\prime}\right|+|Z-P|<2 \sigma^{\prime} r_{k}$.

For $Z \in L_{k}(Q) \cap B(Q, r)$, there exists $Z^{\prime} \in L_{k}(Q) \cap B\left(Q, r-\sigma^{\prime} r_{k}\right)$ so that $\left|Z-Z^{\prime}\right|<\sigma^{\prime} r_{k}$. By (4.30) there exists $P \in \partial \Omega \cap B\left(Q, r_{k}\right)$ so that $\left|Z^{\prime}-P\right|<\sigma^{\prime} r_{k}$. Note that $|Z-P| \leq\left|Z-Z^{\prime}\right|+\left|Z^{\prime}-P\right|<2 \sigma^{\prime} r_{k}$, moreover $|P-Q| \leq\left|P-Z^{\prime}\right|+\left|Z^{\prime}-Q\right|<r$. Thus $P \in \partial \Omega \cap B(Q, r)$. The previous argument ensures that for $Q \in \partial \Omega$ and $r \in(0, \rho)$ there exists an $n$-plane through $Q, L(Q, r)$ so that

$$
\frac{1}{r} D[\partial \Omega \cap B(Q, r), L(Q, r) \cap B(Q, r)] \leq 2 \sigma^{\prime} .
$$

Thus for $\sigma \in\left(0, \frac{\sigma_{\theta^{\prime}}}{2}\right)$ there exists $\varepsilon_{\sigma}>0$ so that if $\varepsilon<\varepsilon_{\sigma}$ and $\sup _{\partial \Omega}|\log h|<\varepsilon$ then $\theta(Q, r) \leq \sigma$ for $r \in(0 ; \rho)$ with $\rho=\sqrt{2} \sqrt{e^{2 \varepsilon}-1} R_{1}$. 
We now focus our attention in the proofs of Lemmas 4.1 and 4.2. As mentioned earlier these are just small variations of results that appear both in [1] and [6], thus we do not present all the details.

Proof of Lemma 4.1. Without loss of generality we may assume that $Q_{0}=0 \in \partial \Omega, \rho=1$ and $\nu=e_{n+1}$. By hypothesis $G \in F(\sigma, 1 ; \tau)$ in $B_{1}=B(0,1)$ in the direction $e_{n+1}, h(Q) \geq e^{-\varepsilon}$ for $\mathcal{H}^{n}$ a.e. $Q \in \partial \Omega$ and $\sup _{B_{1}}|\nabla G| \leq e^{\varepsilon}(1+\tau) \leq e^{\varepsilon}(1+\sigma)$.

This implies that for $\varphi \in C_{0}^{\infty}\left(\mathbb{R}^{n+1}\right), \varphi \geq 0$

$$
\int_{\Omega} G \Delta \varphi \geq e^{-\varepsilon} \int_{\partial \Omega} \varphi d \mathcal{H}^{n}
$$

Let $\eta(Y)=\exp \left(\frac{-9|Y|^{2}}{1-9|Y|^{2}}\right)$ for $|Y|<\frac{1}{3}$ and $\eta(Y)=0$ otherwise. Choose $s_{0}>0$ to be the maximum $s$ so that

$$
B_{1} \cap\{G>0\} \subset D=\left\{X \in B_{1}: x_{n+1}<2 \sigma-s \eta(\bar{x})\right\}
$$

where $X=\left(\bar{x}, x_{n+1}\right)$ with $\bar{x} \in \mathbb{R}^{n} \times\{0\}$ Note that $s_{0} \leq 2 \sigma$. Since $G \in$ $F(\sigma, 1 ; \tau)$ in $B_{1}$ there exists $Z \in \partial D \cap \partial \Omega \cap B\left(0, \frac{1}{3}\right)$. Let $B \subset D^{C}$ be a tangent ball to $D$ at $Z$. Since $\partial D \cap B_{1}$ is smooth and $s_{0} \leq 2 \sigma \leq \sigma_{n}$ for $\sigma_{n}>0$ small we may assume that the radius of $B$ is $\frac{C_{n}}{\sigma_{n}}$. Consider the function $V$ defined by $\Delta V=0$ in $D, V=0$ in $\partial D \cap B_{1}$ and $V=2 \sigma-x_{n+1}$ on $\partial D \backslash B_{1}$. By the maximum principle $V>0$ in $D$ and

$$
G \leq V \text { in } D
$$

as $G \leq V$ on $\partial D$ and $G$ is subharmonic. For $X \in D$ define $F(X)=$ $\left(2 \sigma-x_{n+1}\right)-V(X), F$ is a harmonic function on $D$. Since $Z$ is a smooth point of $\partial D$, standard boundary regularity arguments (see [3, Lemma 6.5]) ensures that $\sup _{X \in \bar{D}}|\nabla F(X)| \leq C \sup _{\bar{D}}|F| \leq C s_{0} \leq C \sigma$. Therefore

$$
-\frac{\partial V}{\partial x_{n+1}}(Z)=1+\frac{\partial F}{\partial x_{n+1}}(Z) \leq 1+C \sigma .
$$

Using (4.35) and noting that $\left|\vec{n}(Z)-e_{n+1}\right| \leq c \sigma$ we have that if $\langle\nabla V, \vec{n}\rangle=\frac{\partial V}{\partial n}$ where $\vec{n}$ denotes the outward unit normal to $\partial D$ then

$$
-\frac{\partial V}{\partial b}(Z) \leq 1+c \sigma+(1+\sigma)\left|\vec{n}-e_{n+1}\right| \leq 1+c \sigma .
$$

Our goal now is to estimate $G$ from below by the linear function $-x_{n+1}$ up to a constant of order $\sigma$. Let $\zeta \in \partial B\left(0, \frac{3}{4}\right) \cap\left\{x_{n+1}<-\frac{1}{2}\right\}$. Consider the function $\omega_{\zeta}$ defined by $\Delta \omega_{\zeta}=0$ in $D \backslash B\left(\zeta, \frac{1}{8}\right), \omega_{\zeta}=0$ on $\partial D \omega_{\zeta}=-x_{n+1}$ on $\partial B\left(\zeta, \frac{1}{8}\right)$. 
The Hopf boundary point lemma ensures that

$$
-\frac{\partial \omega_{\zeta}}{\partial n}(Z) \geq C_{n}>0
$$

Assume that there exists $d>0$ such that $\forall X \in \bar{B}\left(\zeta, \frac{1}{8}\right)$

$$
G(X) \leq V(X)+\sigma d x_{n+1}
$$

The maximum principle would then imply that

$$
G(X) \leq V(X)-d \sigma \omega_{\zeta}(X) \quad \text { in } D \backslash B\left(\zeta, \frac{1}{8}\right) .
$$

Combining Lemma 4.1, (4.36), (4.31), (4.5) and the hypothesis that $\varepsilon \in$ $(0, \sigma)$ we would have

$$
1-\sigma \leq 1-\varepsilon \leq-\frac{\partial V}{\partial n}(Z)-d \sigma \frac{\partial \omega_{\zeta}}{\partial n}(Z) \leq 1+C \sigma-C_{n} d \sigma
$$

which is a contradiction for $d$ large. Thus fr $d$ large enough (depending on $n)$ there are points $X_{\zeta} \in B\left(\zeta, \frac{1}{8}\right)$ such that

$$
G\left(X_{\zeta}\right) \geq V\left(X_{\zeta}\right)+d \sigma\left(X_{\zeta}\right)_{n+1}
$$

Let $X \in B\left(X_{\zeta}, \frac{1}{4}\right)$ then noting that $V(X) \geq-x_{n+1}$ for $X \in D$, using the fact that $\sup _{B_{1}}|\nabla G| \leq e^{\varepsilon}(1+\sigma)$ and (4.41) we have for $\sigma_{n}$ small enough

$$
\begin{aligned}
G(X) & \geq G\left(X_{\zeta}\right)-\sup _{B\left(\zeta, \frac{1}{4}\right)}|\nabla G|\left|X-X_{\zeta}\right| \\
& \geq V\left(X_{\zeta}\right)+d \sigma\left(X_{\zeta}\right)_{n+1}-\frac{1}{4}(1+\sigma) e^{\varepsilon} \\
& \geq-\left(X_{\zeta}\right)_{n+1}+d \sigma\left(X_{\zeta}\right)_{n+1}-\frac{1}{4}(1+\sigma) e^{\varepsilon} \\
& \geq \frac{5}{8}-\frac{7}{8} d \sigma-\frac{1}{4}(1+\sigma) e^{\varepsilon} \\
& \geq \frac{5}{8}-\frac{7}{8} d \sigma-\frac{1}{4}(1+\sigma) e^{\varepsilon}>0
\end{aligned}
$$

for $\sigma<\sigma_{n}$. Since $G(X)>0$ for $X \in \overline{B\left(X_{\zeta}, \frac{1}{4}\right)}, G$ is harmonic on $B\left(X_{\zeta}, \frac{1}{4}\right)$ and so is $V-G$. Moreover

$$
V-G \geq 0 \quad \text { on } \quad B\left(X_{\zeta}, \frac{1}{4}\right) \supset B\left(\zeta, \frac{1}{8}\right) .
$$


Harnack's inequality combined with (4.41) yields

$$
(V-G)(\xi) \leq C_{n}(V-G)\left(X_{\zeta}\right) \leq-C d \sigma\left(X_{\zeta}\right)_{n+1} \leq C \sigma
$$

and

$$
G(\zeta) \geq V(\zeta)-C \sigma \geq-\zeta_{n+1}-C \sigma .
$$

For $X \in D \cap B\left(0, \frac{1}{2}\right), X=\zeta+t x_{n+1}$ for some $\zeta \in \partial B\left(0, \frac{3}{4}\right) \cap\left\{x_{n+1}<-\frac{1}{2}\right\}$ then (4.44) implies that

$$
G(X) \geq G(\zeta)-(1+\sigma) e^{\varepsilon} t \geq-\left(\zeta_{n+1}+t\right)-C \sigma
$$

since $G \in F(\sigma, 1 ; \tau)$ in $B_{1}$ in direction $e_{n+1}$, inequality (4.45) ensures that $G \in F(2 \sigma, C \sigma ; \tau)$ in $B\left(0, \frac{1}{2}\right)$ in direction $e_{n+1}$.

Lemma 4.2 is proved by contradiction, using a non-homogeneous blowup. Assume that Lemma 4.2 does not hold. There exists $\theta_{0} \in(0,1)$ such that for every $\eta>0$ (later we specify one) and every non-negative decreasing sequence $\left\{\sigma_{j}\right\}$ there is a sequence $\left\{\tau_{j}\right\}$ with $\tau_{j} \sigma_{j}^{-2} \rightarrow 0$ so that

$$
G \in F\left(\sigma_{j}, \sigma_{j} ; \tau_{j}\right) \text { in } B\left(Q_{j}, \rho_{j}\right) \text { in direction } \nu_{j}
$$

but

$$
G \notin F\left(\theta_{0} \sigma_{j}, 1 ; \tau_{j}\right) \text { in } B\left(Q_{j}, \eta \rho_{j}\right) .
$$

Since the estimate in Lemma 4.2 is to hold uniformly on compact sets we assume that for each $j \in \mathbb{N}, Q_{j} \in K$ and that $\lim _{j \rightarrow \infty} Q_{j}=Q_{0} \in K Q_{0} \neq 0$ where $K$ is a fixed compact set in $\mathbb{R}^{n+1}$.

Note that if $G \in F(\sigma, \sigma ; \tau)$ in $B(Q, \rho)$ in direction $\nu$ then $G \in F(4 \sigma, 4 \sigma ; \tau)$ in $B\left(P, \frac{\rho}{2}\right)$ in direction $\nu$ for every $P \in \partial \Omega \cap B\left(Q, \frac{P}{2}\right)$. Let $R_{j}$ be the rotation which maps $\mathbb{R}_{+}^{n+1}$ onto $\left\{(x, t)=x+t \nu_{j}: x \in\left\langle\nu_{j}\right\rangle^{\perp} ; t \geq 0\right\}$. Let

$$
\Omega_{j}=\rho_{j}^{-1} R_{j}^{-1}\left(\Omega-Q_{j}\right), \quad \partial \Omega_{j}=\rho_{j}^{-1} R_{j}^{-1}\left(\partial \Omega-Q_{j}\right) .
$$

Define

$$
G_{j}(X)=\frac{1}{\rho_{j} h\left(Q_{j}\right)} G\left(\rho_{j} R_{j} X+Q_{j}\right)
$$

and for $Q \in \partial \Omega_{j}$

$$
h_{j}(Q)=\frac{1}{h\left(Q_{j}\right)} h\left(\rho_{j} R_{j} Q+Q_{j}\right) .
$$

Note that $G_{j}$ is a positive multiple of the Green function of $\Omega_{j}$ with pole $-\rho_{j}^{-1} R_{j}^{-1} Q_{j}$. Note that $\left|\rho_{j}^{-1} R_{j}^{-1} Q_{j}\right| \geq \frac{\left|Q_{0}\right|}{2 \rho_{j}}$ for $j$ large enough. 
Thus for $\varphi \in C_{c}^{\infty}\left(\mathbb{R}^{n+1}\right)$ and $j$ large enough so support $\varphi \subset B\left(0, \frac{\left|Q_{0}\right|}{4 \rho_{j}}\right)$ we have

$$
\int_{\Omega_{j}} G_{j} \Delta \varphi d X=\int_{\partial \Omega_{j}} \varphi h_{j} d \mathcal{H}^{n}
$$

with

$$
\sup _{B(0,1)}\left|\nabla G_{j}\right| \leq 1+\tau_{j} \text { and } \operatorname{osc}_{B(0,1)} h_{j} \leq \tau_{j} \text { with } h_{j}(0)=1 .
$$

Moreover

$$
G_{j} \in F\left(\sigma_{j}, \sigma_{j} ; \tau_{j}\right) \text { in } B(0,1) \text { in direction } e_{n+1}
$$

but

$$
G_{j} \notin F\left(\theta_{0} \sigma_{j}, 1 ; \tau_{j}\right) \text { in } B(0, \eta)
$$

with $\sigma_{j} \rightarrow 0$ and $\tau_{j} \sigma_{j}^{-2} \rightarrow 0$ as $j \rightarrow \infty$.

We define sequences of scaled height functions (in the direction $e_{n+1}$ ) corresponding to $\partial \Omega_{j}$. We prove that this sequence converges to a subharmonic Lipschitz function, and use this information to contradict (4.53) for $j$ large enough. For $y \in B(0,1) \cap \mathbb{R}^{n} \times\{0\}=B^{\prime}$ define

$$
f_{j}^{+}(Y)=\sup \left\{h:\left(y_{1} \sigma_{j} h\right) \in \partial\left\{G_{j}>0\right\}\right\} \leq 1
$$

and

$$
f_{j}^{-}(Y)=\inf \left\{h:\left(y, \sigma_{j} h\right) \in \partial\left\{G_{j}>0\right\}\right\} \geq-1
$$

Lemma 4.4 (Non-homogeneous blow up (Lemma 7.3 in [1])) There exists a subsequence $k_{j}$ such that for $y \in B^{\prime}$

$$
f(y)=\limsup _{\substack{k_{j} \rightarrow \infty \\ z \rightarrow y}} f_{k_{j}}^{+}(z)=\liminf _{\substack{k_{j} \rightarrow \infty \\ z \rightarrow y}} f_{k_{j}}^{-}(z) .
$$

Corollary 4.1 (Corollary 7.4 in [1]) The function $f$ that appears in (4.56) is a continuous function in $B^{\prime}, f(0)=0$; and $f_{k_{j}}^{+}$and $f_{k_{j}}^{-}$converge uniformly to $f$ on compact sets of $B^{\prime}$.

The proofs of Lemma 4.4 and Corollary 4.1 are identical to those that appear in [1] or [6], thus we omit them here.

Lemma 4.5 (Lemma 7.5 in [1]) The function $f$ introduced in Lemma 4.4 is subharmonic in $B^{\prime}$. 
Proof. This proof is done by contradiction. Assuming that $f$ is not subharmonic in $B^{\prime}$ we contradict the fact that $\sigma_{j}^{-2} \tau_{j} \rightarrow 0$ as $j \rightarrow \infty$. In fact if $f$ is not subharmonic in $B^{\prime}$ there exists $y_{0} \in B^{\prime}$ and $\rho>0$ so that $B^{\prime}\left(y_{0}, \rho\right) \subset B^{\prime}$ and

$$
f\left(y_{0}\right)>f_{\partial B^{\prime}\left(y_{0}, \rho\right)} f(x) d x
$$

Let

$$
\varepsilon_{0}=\frac{f\left(y_{0}\right)-\gamma_{\partial B^{\prime}\left(y_{0}, \rho\right)} f(x) d x}{2}
$$

Let $g$ be the solution to the Dirichlet problem

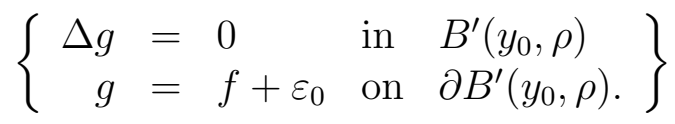

Note that

$$
\begin{aligned}
f<g \text { on } \partial B^{\prime}\left(y_{0}, \rho\right), \text { and } \\
g\left(y_{0}\right)=f_{\partial B^{\prime}\left(y_{0}, \rho\right)} g(x) d x=f_{\partial B^{\prime}\left(y_{0}, \rho\right)} f(x) d x+\varepsilon_{0} \\
g\left(y_{0}\right)=\frac{1}{2}\left\{f\left(y_{0}\right)+f_{\partial B^{\prime}\left(y_{0}, \rho\right)} f(x) d x\right\} \\
g\left(y_{0}\right)<f\left(y_{0}\right) .
\end{aligned}
$$

Summarizing, we have the following picture.

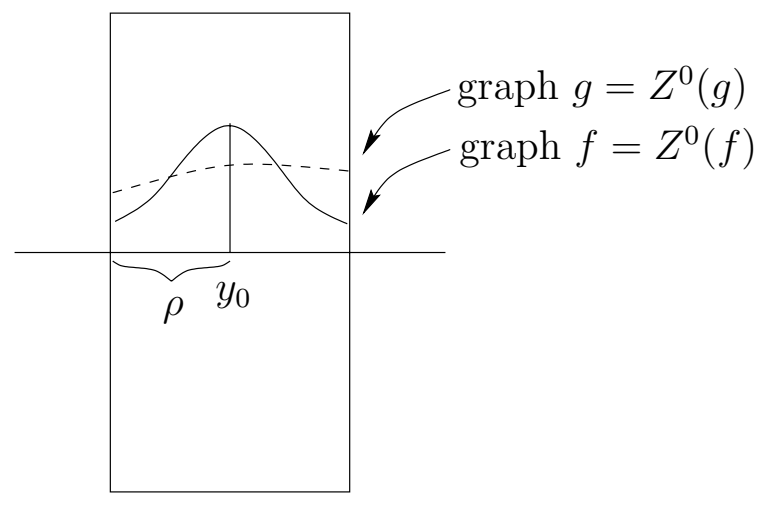

$$
\left\{\begin{array}{rlrl}
\Delta g & =0 & \text { in } \quad B^{\prime}\left(y_{0}, \rho\right) \\
g & >f & \text { in } & \partial B^{\prime}\left(y_{0}, \rho\right) \\
g\left(y_{0}\right) & <f\left(y_{0}\right) & &
\end{array}\right.
$$


The main idea of the proof is to compare the $n$-dimensional Hausdorff measure of $\partial\left\{G_{k_{j}}>0\right\}$ on the cylinder $B^{\prime}\left(y_{0}, \rho\right) \times(-1,1)$ to that of the graph of $\sigma_{k_{j}} g$ on the same cylinder to obtain a contradiction from an estimate on the size of the area enclosed by these 2 surfaces. In order to simplify the notation we relabel the sequences that appear in Lemma 4.4. We also introduce some new definitions.

Let $Z=B^{\prime}\left(y_{0}, \rho\right) \times \mathbb{R}$ be the infinite cylinder. For $\phi$ defined on $\mathbb{R}^{n}$ define

$$
\begin{aligned}
Z^{+}(\phi) & =\{(y, h) \in Z: h>\phi(y)\} \\
Z^{-}(\phi) & =\{(y, h) \in Z: h<\phi(y)\} \\
Z^{0}(\phi) & =\{(y, h) \in Z: h=\phi(y)\} .
\end{aligned}
$$

We may assume that for $k$ large enough

$$
\mathcal{H}^{n}\left(Z^{0}\left(\sigma_{k} g\right) \cap \partial\left\{G_{k}>0\right\}\right)=0 .
$$

It might be necessary to modify $g$ above by adding a suitable constant which can be chosen as small as one wants. In particular the function $g$ would still satisfy (4.60) and (4.61).

Claim 1 For $k$ large enough

$$
\mathcal{H}^{n}\left(Z^{+}\left(\sigma_{k} g\right) \cap \partial\left\{G_{k}>0\right\}\right) \leq \frac{1+\tau_{k}}{1-\tau_{k}} \mathcal{H}^{n}\left(Z^{0}\left(\sigma_{k} g\right) \cap\left\{G_{k}>0\right\}\right) .
$$

Claim 2 Let $E_{k}=\left\{G_{k}>0\right\} \cap Z^{-}\left(\sigma_{k} g\right)$. $E_{k}$ is a set of locally finite perimeter and

$$
\begin{aligned}
\mathcal{H}^{n}\left(Z \cap \partial^{*} E_{k}\right) \leq \mathcal{H}^{n}\left(\partial\left\{G_{k}>0\right\} \cap Z^{+}\left(\sigma_{k} g\right)\right) \\
+\mathcal{H}^{n}\left(\left\{G_{k}=0\right\} \cap Z^{0}\left(\sigma_{k} g\right)\right) .
\end{aligned}
$$

Here $\partial^{*} E_{k}$ denotes the reduced boundary of $E_{k}$.

Claim 3 There exists a constant $C>0$ such that

$$
\mathcal{H}^{n}\left(Z \cap \partial^{*} E_{k}\right) \geq \mathcal{H}^{n}\left(Z^{0}\left(\sigma_{k} g\right)\right)+C \sigma_{k}^{2} \rho^{n} .
$$

Before proving the claims we indicate how combining inequalities (4.66), (4.67) and (4.68) we obtain a contradiction. Combining (4.66), (4.67) and (4.68) and using (4.65) we have

$$
\begin{aligned}
& \mathcal{H}^{n}\left(Z^{0}\left(\sigma_{k} g\right)\right)+C \sigma_{k}^{2} \rho^{n} \leq \mathcal{H}^{n}\left(Z \cap \partial^{*} E_{k}\right) \\
& \leq \mathcal{H}^{n}\left(\partial\left\{G_{k}>0\right\} \cap Z^{+}\left(\sigma_{k} g\right)+\mathcal{H}^{n}\left(\left\{G_{k}=0\right\} \cap Z^{-}\left(\sigma_{k} g\right)\right)\right. \\
& \leq \frac{1+\tau_{k}}{1-\tau_{k}} \mathcal{H}^{n}\left(Z^{0}\left(\sigma_{k} g\right) \cap\left\{G_{k}>0\right\}\right)+\mathcal{H}^{n}\left(\left\{G_{k}=0\right\} \cap Z^{0}\left(\sigma_{k} g\right)\right) \\
& \leq \frac{2 \tau_{k}}{1-\tau_{k}} \mathcal{H}^{n}\left(Z^{0}\left(\sigma_{k} g\right) \cap\left\{G_{k}>0\right\}\right)+\mathcal{H}^{n}\left(Z^{0}\left(\sigma_{k} g\right)\right)
\end{aligned}
$$


which implies

$$
\begin{aligned}
C \sigma_{k}^{2} \rho^{n} & \leq \frac{2 \tau_{k}}{1-\tau_{k}} \mathcal{H}^{n}\left(Z^{0}\left(\sigma_{k} g\right) \cap\left\{G_{k}>0\right\}\right) \\
& \leq \frac{2 \tau_{k}}{1-\tau_{k}} \int_{B_{\rho^{\prime}\left(y_{0}\right)}} \sqrt{1+\sigma_{k}^{2}|\nabla g|^{2}}
\end{aligned}
$$

For $\tau_{k}<\frac{1}{2}$ and $\sigma_{k}<1(4.70)$ yields $C \sigma_{k}^{2} \leq C^{\prime} \tau_{k}$ which contradicts the fact that $\tau_{k} \sigma_{k}^{-2} \rightarrow 0$ as $k \rightarrow \infty$. Thus we conclude that $f$ is subharmonic in $B^{\prime}$.

Proof of Claim 1. Since $h_{k}(0)=1$ and $\operatorname{osc}_{B(0,1)} h_{k} \leq \tau_{k}$ we have that

$$
\begin{aligned}
\mathcal{H}^{n}\left(Z^{+}\left(\sigma_{k} g\right) \cap \partial\left\{G_{k}>0\right\}\right) & =\int_{Z^{+}\left(\sigma_{k} g\right) \cap \partial\left\{G_{k}>0\right\}} d \mathcal{H}^{n} \\
& \leq \frac{1}{1-\tau_{k}} \int_{Z^{+}\left(\sigma_{k} g\right) \cap \partial\left\{G_{k}>0\right\}} h_{k} d \mathcal{H}^{n}
\end{aligned}
$$

For $\varphi \in C_{c}^{\infty}\left(\mathbb{R}^{n+1}\right)$ and $k$ large enough we have

$$
-\int_{\left\{G_{k}>0\right\}} \nabla G_{k} \nabla \varphi=\int_{\partial\left\{G_{k}>0\right\}} \varphi h_{k} d \mathcal{H}^{n} .
$$

Letting $\varphi \rightarrow \chi_{Z^{+}\left(\sigma_{k} g\right)},(4.72)$ yields

$$
-\int_{\left\{\overline{G_{k}>0}\right\} \cap \partial Z^{+}\left(\sigma_{k} g\right)} \nabla G_{k} \cdot \nu=\int_{\partial\left\{G_{k}>0\right\} \cap Z^{+}\left(\sigma_{k} g\right)} h_{k} d \mathcal{H}^{n}
$$

where $\nu$ denotes the outward pointing unit normal. Combining (4.65), (4.71), (4.73) and (4.51) we have that

$$
\begin{aligned}
\mathcal{H}^{n}\left(Z^{+}\left(\sigma_{k} g\right) \cap \partial\left\{G_{k}>0\right\}\right) & \leq \frac{1}{1-\tau_{k}} \int_{\left\{G_{k}>0\right\} \cap \partial Z^{+}\left(\sigma_{k} g\right)}\left|\nabla G_{k}\right| \\
& \leq \frac{1+\tau_{k}}{1-\tau_{k}} \mathcal{H}^{n}\left(\left\{G_{k}>0\right\} \cap Z^{0}\left(\sigma_{k} g\right)\right) .
\end{aligned}
$$

The proof of Claim 2 is straightforward. The proof of Claim 3 is identical to the one that appears in either [1] or [6], thus we do not present it here.

To obtain the desired contradiction we need to prove that $f$ is Lipschitz. This proof relies on the following lemma which claims that $f$ converges to its average faster than linearly in an integral sense. 
Lemma 4.6 (Lemma 7.6 in [1]) There is a constant $C=C(n)>0$ such that for $y \in B_{1 / 2}^{\prime}=B\left(0, \frac{1}{2}\right) \cap \mathbb{R}^{n} \times\{0\}$

$$
0 \leq \int_{0}^{\frac{1}{4}} \frac{1}{r^{2}}\left(f_{y, r}-f(y)\right) d r \leq C
$$

where

$$
f_{y, r}=\int_{\partial B^{\prime}(y, r)} f d \mathcal{H}^{n-1}
$$

Proof. The proof is very similar to the ones that appear in [1] and [6]. Nevertheless since the minor differences are technically important we sketch the proof here pointing out how to overcome the difficulties that arise in this situation. For the complete details we refer the reader to [1] or [6]. Without loss of generality we may assume that $y=0$. Since $f(0)=0$ it is enough to show

$$
0 \leq \int_{0}^{\frac{1}{4}} \frac{1}{r^{2}} \int_{\partial B_{r}^{\prime}} f d \mathcal{H}^{n-1} \leq C
$$

where $B_{r}^{\prime}=B^{\prime}(0, r)$ and $C$ only depends on $n$ since $f$ is subharmonic (see Lemma 4.5) then for $r \in\left(0, \frac{1}{2}\right), f(0) \leq \oint_{\partial B_{r}^{\prime}} f d \mathcal{H}^{n-1}$ which proves the first inequality.

Let $h>2 \sigma_{j}$ be small and let $G_{h}$ denote the Green function of $B\left(0, \frac{1}{2}\right) \cap$ $\left\{x_{n+1}<0\right\}$ with pole $-h e_{n+1}$. By reflection $G_{h}$ can be extended to a smooth function on $B\left(0, \frac{1}{2}\right) \backslash\left\{ \pm h e_{n+1}\right\}$ with $G_{h}\left(\bar{x}, x_{n+1}\right)=-G\left(\bar{x},-x_{n+1}\right)$ for $x_{n+1}>0$. For $j$ large let $G_{h}^{j}(X)=G_{n}\left(X+\sigma_{j} e_{n+1}\right)$ be defined on $B\left(\frac{1}{2},-\sigma_{j} e_{n+1}\right) \backslash\left\{\left(\sigma_{j} \pm h\right) e_{n+1}\right\}$. We denote by $B_{1 / 2}=B\left(0, \frac{1}{2}\right)$ and by $B_{1 / 2}^{j}=$ $B\left(\frac{1}{2} ;-\sigma_{j} e_{n+1}\right)$. We may assume that $\mathcal{H}^{n}\left(\partial B_{1 / 2}^{j} \cap \partial\left\{G_{j}>0\right\}\right)=0$. Green's formula ensures that

$$
-\int_{B_{1 / 2}^{j}}\left\langle\nabla G_{j}, \nabla G_{h}^{j}\right\rangle=\int_{\partial B_{1 / 2}^{j}} G_{j} \partial_{\nu} G_{h}^{j}-G_{j}\left(-\left(h+\sigma_{j}\right) e_{n+1}\right),
$$

where $\partial_{\nu} G_{h}^{j}=\left\langle\nabla G_{h}^{j}, \nu\right\rangle$, and $\nu$ denotes the inward pointing unit normal to $\partial B_{1 / 2}^{\prime}$. On the other hand

$$
-\int_{\partial B_{1 / 2}^{j}}\left\langle\nabla G_{j}, \nabla G_{h}^{j}\right\rangle=\int_{\partial\left\{G_{j}>0\right\} \cap B_{1 / 2}^{j}} h_{j} G_{h}^{j} d \mathcal{H}^{n} .
$$


Let $\nu_{j}$ denote the inward point unit normal to $\partial \Omega_{j}=\partial\left\{G_{j}>0\right\}$ then by Green's formula we have

$$
\begin{aligned}
\int_{B_{1 / 2}^{j} \cap \partial\left\{G_{j}>0\right\}}\left\langle G_{h}^{j} e_{n+1}-x_{n+1} \nabla G_{h}^{j}, \nu_{j}\right\rangle d \mathcal{H}^{n} \\
=\left(\sigma_{j}+h\right)+\int_{\partial B_{1 / 2}^{j} \cap\left\{G_{j}>0\right\}} x_{n+1} \partial_{\nu} G_{h}^{j} .
\end{aligned}
$$

Combining (4.78), (4.79) and (4.80) we obtain

$$
\begin{aligned}
& \int_{B_{1 / 2}^{j} \cap \partial\left\{G_{j}>0\right\}} x_{n+1} \partial_{\nu_{j}} G_{h}^{j} d \mathcal{H}^{n} \\
= & \int_{B_{1 / 2} \cap \partial\left\{G_{j}>0\right\}}\left(h_{j}+\left\langle e_{n+1}, \nu_{j}\right\rangle\right) G_{h}^{j} d \mathcal{H}^{n} \\
& -\int_{\partial B_{1 / 2}^{j} \cap\left\{G_{j}>0\right\}}\left(x_{n+1}+G_{j}\right) \partial_{\nu} G_{h}^{j}+G_{j}\left(-\left(h+\sigma_{j}\right) e_{n+1}\right)-\left(\sigma_{j}+h\right) \\
= & \int_{B_{1 / 2} \cap \partial\left\{G_{j}>0\right\}}\left(\frac{h_{j}}{1-\tau_{j}}+\left\langle e_{n+1}, \nu_{j}\right\rangle\right) G_{h}^{j} d \mathcal{H}^{n} \\
& -\tau_{j} \int_{B_{1 / 2} \cap \partial\left\{G_{j}>0\right\}} h_{j} G_{h}^{j} d \mathcal{H}^{n}+G_{j}\left(-\left(h+\sigma_{j}\right) e_{n+1}\right)-\left(\sigma_{j}+h\right) \\
& -\int_{\partial B_{1 / 2}^{j} \cap\left\{G_{j}>0\right\}}\left(x_{n+1}+G_{j}\right) \partial_{\nu} G_{n}^{j} \\
= & \int_{B_{1 / 2} \cap \partial\left\{G_{j}>0\right\}}\left(\frac{h_{j}}{1-\tau_{j}}+\left\langle e_{n+1}, \nu_{j}\right\rangle\right) G_{h}^{j} d \mathcal{H}^{n} \\
& +\left(1+\tau_{j}\right) G_{j}\left(-\left(h+\sigma_{j}\right) e_{n+1}\right)-\left(\sigma_{j}+h\right) \\
& -\int_{\partial B_{1 / 2}^{j} \cap\left\{G_{j}>0\right\}}\left(x_{n+1}+G_{j}\left(1+\tau_{j}\right)\right) \partial_{\nu} G_{h}^{j} .
\end{aligned}
$$

Since $\sigma_{j}-h<-\sigma_{j}$ and $G_{j} \in F\left(\sigma_{j}, \sigma_{j} ; \tau_{j}\right)$ in $B(0,1)$ in direction $e_{n+1}$, then $G_{h}^{j} \leq 0$ on $\partial\left\{G_{j}>0\right\} \cap B_{1 / 2}^{j}$. Furthermore since $h_{j}(0)=1$, by $(4.51)$ $h_{j} \geq 1-\tau_{j}$ on $B_{1 / 2}^{j} \cap \partial\left\{G_{j}>0\right\}$.

Thus

$$
\int_{B_{1 / 2} \cap \partial\left\{G_{j}>0\right\}}\left(\frac{h_{j}}{1-\tau_{j}}+\left\langle e_{n+1}, \nu_{j}\right\rangle\right) G_{h}^{j} \leq 0 .
$$

Since $G_{j}(0)=0,(4.51)$ ensures that

$$
\left|G_{j}\left(-\left(h+\sigma_{j}\right) e_{n+1}\right)\right| \leq \sup _{B(0,1)}\left|\nabla G_{j}\right|\left(h+\sigma_{j}\right) \leq\left(1+\tau_{j}\right)\left(h+\sigma_{j}\right) .
$$


Hence

$$
\left(1+\tau_{j}\right) G_{j}\left(-\left(h+\sigma_{j}\right) e_{n+1}\right)-\left(\sigma_{j}+h\right) \leq 3 \tau_{j}\left(h+\sigma_{j}\right)
$$

Since $\left\{G_{j}>0\right\} \subset\left\{x_{n+1}<\sigma_{j}\right\}$, by (4.51) for $x_{n+1} \leq \sigma_{j}$ we have in $B(0,1)$

$$
G_{j}\left(\bar{x}, x_{n+1}\right)=\left|G_{j}\left(\bar{x}, x_{n+1}\right)-G_{j}\left(\bar{x}, \sigma_{j}\right)\right| \leq\left(1+\tau_{j}\right)\left(\sigma_{j}-x_{n+1}\right)
$$

which yields

(4.86) $x_{n+1} \leq x_{n+1}+\left(1+\tau_{j}\right) G_{j}\left(\bar{x}, x_{n+1}\right) \leq\left(1-\left(1+\tau_{j}\right)^{2}\right) x_{n+1}+\left(1+\tau_{j}\right)^{2} \sigma_{j}$.

Thus

$$
0 \leq x_{n+1}+\left(1+\tau_{j}\right) G_{j}\left(\bar{x}, x_{n+1}\right) \leq\left(1+\tau_{j}\right)^{2} \sigma_{j} \quad \text { for } x_{n+1} \in\left[0, \sigma_{j}\right]
$$

(4.88) $-\sigma_{j} \leq x_{n+1}+\left(1+\tau_{j}\right) G_{j}\left(\bar{x}, x_{n+1}\right) \leq\left(1+\tau_{j}\right) \sigma_{j} \quad$ for $x_{n+1} \in\left[-\sigma_{j}, 0\right]$.

Since $G_{j} \in F\left(\sigma_{j}, \sigma_{j} ; \tau_{j}\right)$ in $B(0,1)$ in direction $e_{n+1}$ with $h_{j}(0)=1$ then

$$
\begin{aligned}
& x_{n+1}+\left(1+\tau_{j}\right) G_{j}\left(\bar{x}, x_{n+1}\right) \geq x_{n+1}+\left(1+\tau_{j}\right)\left(-x_{n+1}-\sigma_{j}\right) \\
& \geq-\tau_{j} x_{n+1}-\sigma_{j}\left(1+\tau_{j}\right) \geq-\sigma_{j}\left(1+\tau_{j}\right) \quad \text { for } x_{n+1} \leq-\sigma_{j}
\end{aligned}
$$

We combine the fact that $\partial_{\nu} G_{h}^{j} \geq 0$ with (4.87), (4.88) and (4.89) and obtain that

$$
\begin{aligned}
-\int_{\partial B_{1 / 2}^{j} \cap\left\{G_{j}>0\right\}} & \left(x_{n+1}+\left(1+\tau_{j}\right) G_{j}\right) \partial_{\nu} G_{h}^{j} \\
& \leq \sigma_{j}\left(1+\tau_{j}\right) \int_{\partial B_{1 / 2}^{j} \cap\left\{G_{j}>0\right\} \cap\left\{x_{n+1}<0\right\}} \partial_{\nu} G_{n}^{j} .
\end{aligned}
$$

Combining (4.81), (4.82), (4.84), (4.90), the fact that $\sigma_{j}^{-2} \tau_{j} \leq 1$ for $j$ large enough, and that $1 \geq h>2 \sigma_{j}$ we conclude that

$$
\text { (4.91) } \frac{1}{\sigma_{j}} \int_{B_{1 / 2}^{j} \cap \partial\left\{G_{j}>0\right\}} x_{n+1} \partial_{\nu_{j}} G_{h}^{j} \leq 9 \sigma_{j}+2 \int_{\partial B_{1 / 2}^{j} \cap\left\{G_{j}>0\right\} \cap\left\{x_{n+1}<0\right\}} \partial_{\nu} G_{n}^{j} .
$$

Thus

$$
\text { (4.92) } \limsup _{j \rightarrow \infty} \frac{1}{\sigma_{j}} \int_{B_{1 / 2}^{j} \cap \partial\left\{G_{j}>0\right\}} x_{n+1} \partial_{\nu_{j}} G_{h}^{j} \leq 2 \int_{\partial B_{1 / 2} \cap\left\{x_{n+1} \leq 0\right\}} \partial_{\nu} G_{h} \leq C h .
$$

The rest of the argument is identical to the one that appears in [6] in the proof of Lemma 0.9 . 
Lemma 4.7 (Lemmata 7.7 and 7.8 in [1]) The function $f$ introduced in Lemma 4.4 is Lipschitz in $B_{1 / 16}^{\prime}$ with Lipschitz constant that only depends on $n$. Furthermore there exists a large constant $C=C(n)>0$ such that for any given $\theta \in(0,1)$ there exists $\eta=\eta(\theta)>0$ and $l \in \mathbb{R}^{n} \times\{0\}$ with $|l| \leq c$ so that

$$
f(y) \leq\langle l, y\rangle+\frac{\theta}{2} \eta \text { for } y \in B_{\eta}^{\prime}
$$

The proof of this lemma basically appears in [1] and [6]

Now we indicate how the last 2 lemmata yield a contradiction in the proof of Lemma 4.2. Recall that by assuming that the statement in Lemma 4.2 is false we can construct sequences of function $\left\{G_{j}\right\}$ and $\left\{h_{j}\right\}$ satisfying (4.50), (4.51), (4.52) and (4.53). From them as in (4.54), (4.55) and Lemmas 4.4, $4.5,4.6$ and 4.7 we can produce a subharmonic Lipschitz function $f$ on $B_{1 / 16}^{\prime}$ satisfying (4.93). Recall that by Lemma 4.4 and Corollary $4.1 f$ is uniform limit of the functions $f_{j}^{+}$defined in (4.54). Therefore Lemma 4.7 yields that for $\theta \in(0,1)$ there exists $\eta>0$ so that for $j$ large enough

$$
f_{j}^{+}(y) \leq\langle l, y\rangle+\theta \eta \text { for } y \in B_{\eta}^{\prime},
$$

which by definition means that

$$
G_{j}(X)=0 \text { for } X=\left(\bar{x}, x_{n+1}\right) \in B(0, \eta) \text { with } x_{n+1}>\sigma_{j}\langle l, \bar{x}\rangle+\theta \eta \sigma_{j} .
$$

Let $\bar{\nu}=\left(1+\sigma_{j}^{2}|l|^{2}\right)^{-1 / 2}\left(-\sigma_{j} l, 1\right)(4.95)$ implies that

$$
G_{j}(X)=0 \text { for } X \in B(0, \eta) \text { with }\langle X, \bar{\nu}\rangle \geq \frac{\theta \eta \sigma_{j}}{\left(1+\sigma_{j}^{2}|l|^{2}\right)^{1 / 2}} \geq 2 \theta \eta \sigma_{j}
$$

for $j$ large enough. But (4.51) and (4.96) state that $G_{j} \in F\left(2 \theta \eta_{j}, 1 ; \tau_{j}\right)$ in $B(0, \eta)$ in direction $\bar{\nu}$. This contradicts statement (4.53) in the case that $\theta=\frac{\theta_{0}}{2}$, which concludes the proof of Lemma 4.2 and thus that of the Theorem 2.1.

\section{Applications}

Lemma 5.1 Assume that $\Omega \subset \mathbb{R}^{n+1}$ satisfies (2.9). Then there exist $\varepsilon_{0}>0$ and $r_{0}>0$ such that if

$$
\sup _{\partial \Omega}|\log h|<\varepsilon_{0}
$$

then for $Q \in \partial \Omega$ and $r \in\left(0, r_{0}\right)$

$$
C_{n}^{-1} r^{n} \leq \mathcal{H}^{n}(\partial \Omega \cap B(Q, r)) \leq C_{n} r^{n},
$$

where $C_{n}$ is a constant that only depends on $n$, i.e. $\partial \Omega$ is Ahlfors regular. 
Proof. Let $\sigma \in\left(0, \frac{1}{4}\right)$ be small enough in Theorem 2.1 then there exists $\varepsilon_{1}>0$ such that if $\sup _{\partial \Omega}|\log h|<\varepsilon_{1}$, then $\partial \Omega$ is $\sigma$-Reifenberg flat. This ensures that there exists $\rho_{1}>0$ so that for $Q \in \partial \Omega$ and $r<\rho_{1}$

$$
\mathcal{H}^{n}(\partial \Omega \cap B(Q, r)) \geq(1+\sigma)^{-1} \omega_{n} r^{n} \geq \frac{1}{2} \omega_{n} r^{n}
$$

(for the proof see Remark 2.2 in [7]). By Lemma 4.3 there exists $0<\varepsilon_{2}<\varepsilon_{1}$ so that if $\sup _{\partial \Omega}|\log h|<\varepsilon$ with $0<\varepsilon<\varepsilon_{2}$ there exists $\rho_{\varepsilon}=\rho>0$ such that for $Q \in \partial \Omega, G \in F\left(\sigma, \sigma ;\left(e^{2 \varepsilon}-1\right)^{1 / 4}\right)$ in $B\left(Q, \rho_{\varepsilon}\right)$. Thus in particular for $r<\min \left\{\rho_{\varepsilon}, \rho_{1}\right\}$

$$
\sup _{B(Q, r)}|\nabla G| \leq h(Q)\left(1+\left(e^{2 \varepsilon}-1\right)^{1 / 4}\right) \leq e^{\varepsilon}\left(1+\left(e^{2 \varepsilon}-1\right)^{1 / 4}\right) .
$$

Hence

$$
\begin{aligned}
\mathcal{H}^{n}(\partial \Omega \cap B(Q, r)) & =\int_{B(Q, r) \cap \partial \Omega} h \frac{1}{h} d \mathcal{H}^{n} \\
& \leq e^{\varepsilon} \int_{B(Q, r) \cap \partial \Omega} h d \mathcal{H}^{n} \leq e^{\varepsilon} \int_{\partial \Omega} \varphi h d \mathcal{H}^{n} \\
& \leq-e^{\varepsilon} \int_{\Omega}\langle\nabla \varphi, \nabla G\rangle d \mathcal{H}^{n+1}
\end{aligned}
$$

for any non-negative $\varphi \in C_{c}^{\infty}\left(\mathbb{R}^{n+1}\right)$ such that $\varphi \equiv 1$ on $B(Q, r)$ and $0 \notin$ support $\varphi$.

In particular if $\varphi$ is chosen so that $\varphi \in C_{c}^{\infty}(B(Q, 2 r))$ for $r<\frac{1}{2} \min \left\{\rho_{\varepsilon}, \rho_{1}\right\}$ and $|\nabla \varphi|<2 / r,(5.4)$ and (5.5) yield for $\varepsilon>0$ small enough

$$
\mathcal{H}^{n}(B(Q, r) \cap \partial \Omega) \leq e^{\varepsilon} \frac{2}{r} e^{\varepsilon}\left(1+\left(e^{2 \varepsilon}-1\right)^{1 / 4}\right) \omega_{n+1} r^{n+1} \leq 4 \omega_{n+1} r^{n} .
$$

Choosing

$$
\varepsilon_{0}=\min \left\{\frac{\varepsilon_{2}}{2}, \frac{1}{4}\right\} \quad \text { and } \quad r_{0}=\frac{1}{2} \min \left\{\rho_{\varepsilon_{0}}, \rho_{1}\right\}
$$

we conclude that (5.2) holds.

Corollary 5.1 Assume that $\Omega \subset \mathbb{R}^{n+1}$ satisfies (2.9). Then given $\delta>0$ small enough there exists $\varepsilon>0$ such that if

$$
\sup _{\partial \Omega}|\log h|<\varepsilon
$$

then $\Omega$ is a $\delta$-Reifenberg flat chord arc domain. 
Proof. By Theorem 2.1, $\partial \Omega$ is $\delta$-Reifenberg flat provided $\varepsilon>0$ is small enough. Since $\Omega$ is bounded and $B\left(0, R_{1}\right) \subset \Omega \subset B\left(0, R_{2}\right)$ it is easy to show that it satisfies the separation property. Therefore $\Omega$ is a $\delta$-Reifenberg flat domain and for $\delta>0$ small enough it is also NTA (see [7]). Moreover if $\varepsilon<\varepsilon_{0}$ Lemma 5.1 ensures that for $r \in\left(0, r_{0}\right)(5.2)$ holds. Since $\Omega$ is bounded it is easy to see that for $r \in(0, \operatorname{diam} \Omega),(5.2)$ also holds with a constant that only depends on $n$, and $\frac{\operatorname{diam} \Omega}{r_{0}}$. Thus $\Omega$ is a chord arc domain.

The crucial information contained in Lemma 5.1 and Corollary 5.1 is that bounded domains which are sets of locally finite perimeter and satisfy (2.9) belong to a family of chord arc domains with uniform constants.

Corollary 5.2 Assume that $\Omega \subset \mathbb{R}^{n+1}$ satisfies (2.9). There exists $\varepsilon_{1}>0$ so that if $\sup _{\partial \Omega}|\log h|<\varepsilon_{1}$ and $\log h \in \mathrm{VMO}(\partial \Omega)\left(\right.$ resp. $\log h \in C^{k, \alpha}(\partial \Omega)$ ) then $\Omega$ is a chord arc domain with vanishing constant (resp. $\Omega$ is a $C^{k+1, \alpha}$ domain).

Proof. By choosing $\varepsilon_{1}>0$ small enough Corollary 5.1 ensures that $\Omega$ is a $\delta$ Reifenberg flat chord arc domain. Choosing $\delta>0$ as in the statement of the Main Theorem in [8] we conclude that if $\log h \in \mathrm{VMO}$ then $\vec{n} \in \operatorname{VMO}(\partial \Omega)$. Choosing $\delta>0$ as in the statement of Alt and Caffarelli's theorem we conclude that if $\log h \in C^{k, \alpha}$ then $\Omega$ is a $C^{k+1, \alpha}$ domain.

Corollary 5.3 Assume that $\Omega \subset \mathbb{R}^{n+1}$ satisfies (2.9). There exists $\varepsilon_{2}>0$ so that it $\sup _{\partial \Omega}|\log h|<\varepsilon_{2}$ and $\log h \in C^{0, \alpha}$ there exists a homeomorphism $\psi: B\left(0, R_{1}\right) \rightarrow \Omega$ where $\psi$ and $\psi^{-1}$ are $C^{1, \alpha}$.

Proof. By the work in [1] and Corollary 5.1 we know that there exists $\delta>0$ and $\varepsilon>0$ depending on $\delta>0$ so that if $\sup _{\partial \Omega}|\log h|<\varepsilon$ and $\log h \in C^{0, \alpha}$ then $\Omega$ is a $C^{1, \alpha}$ domain. Moreover using the proof of Theorem 8.1 in [1] and (4.7) above we conclude that

$$
\left|\vec{n}(Q)-\frac{Q}{|Q|}\right|<\delta
$$

Here $\vec{n}(Q)$ denotes the outward unit normal to $\partial \Omega$. Since $\Omega$ is a bounded $C^{1, \alpha}$ domain there exists $r \in\left(0, \frac{R_{1}}{8}\right)$ so that for $Q \in \partial \Omega \cap B(Q, r)$ can be written as the area below the graph of a $C^{1, \alpha}$ function (with small $C^{1, \alpha}$ norm 1 over the $n$-plane through $Q$ and orthogonal to $\vec{n}(Q)$. Inequality (5.8) guarantees that $\Omega \cap B(Q, r)$ can also be seen as the area below the graph of a $C^{1, \alpha}$ function (with $C^{1, \alpha}$ norm less than $C \delta$ ) over the $n$ plane through $Q$ and orthogonal to $\frac{Q}{|Q|}$. This implies that the spherical 
projection $S: \partial \Omega \rightarrow B\left(0, R_{1}\right) S(Q)=R_{1} \frac{Q}{|Q|}$ is a $1-1$ map. Moreover since $B\left(Q, R_{1}\right) \subset \Omega, S$ is onto and Lipschitz on $\partial \Omega$. In particular $\Omega$ is star shaped with respect to the origin.

Since $S$ is smooth on $\mathbb{R}^{n+1} \backslash B\left(0, \frac{R_{1}}{4}\right)$ and $\partial \Omega$ is a $C^{1, \alpha}$ submanifold it is clear that $S$ is a $C^{1, \alpha}$ map from $\partial \Omega$ onto $B\left(0, R_{1}\right)$, and $S^{-1}$ is a $C^{1, \alpha}$ map from $\partial B\left(0, R_{1}\right)$ onto $\partial \Omega$. For $X \in \Omega \backslash B\left(0, \frac{R_{1}}{4}\right)$ there exists a unique $Q_{X} \in \partial \Omega$ so that $\frac{X}{|X|}=\frac{Q_{X}}{\left|Q_{X}\right|}$. The previous remark ensures that the map that to $X \in \Omega \backslash B\left(0, \frac{R_{1}}{4}\right)$ associates $Q_{X}$ is a $C^{1, \alpha}$ map. Our goal is to construct a homeomorphism $\Phi: \Omega \rightarrow B\left(0, R_{1}\right)$, such that $\Phi$ and $\Phi^{-1}$ are $C^{1, \alpha}$. Let $X \in \Omega$ and define

$$
g(t)= \begin{cases}t & t \in\left[0, \frac{R_{1}}{4}\right] \\ \frac{R_{1}-\left|Q_{X}\right|}{\left(\left|Q_{X}\right|-\frac{R_{1}}{4}\right)^{2}}\left(t-\frac{R_{1}}{4}\right)^{2}+t & \text { for } t \in\left[\frac{R_{1}}{4},\left|Q_{X}\right|\right] .\end{cases}
$$

In particular $g \in C^{1,1}\left(\left[0,\left|Q_{X}\right|\right]\right), g(0)=0$ and $g\left(\left|Q_{X}\right|\right)=R_{1}$. Moreover since $\left|Q_{X}\right| \geq R_{1}$, for $\varepsilon<\frac{1}{64}, g^{\prime}>0$ on $\left[0,\left|Q_{X}\right|\right]$ thus $g$ is $1-1$ and maps $\left[0,\left|Q_{X}\right|\right]$ onto $\left[0, R_{1}\right]$. For $X \in \Omega$ define

$$
\Phi(X)=
$$

$$
=g(|X|) \frac{X}{|X|}- \begin{cases}X & \text { for } X \in B\left(0, \frac{R_{1}}{4}\right) \\ \left(\frac{R_{1}-\left|Q_{X}\right|}{\left(\left|Q_{X}\right|-\frac{R_{1}}{4}\right)^{2}}\left(|X|-\frac{R_{1}}{4}\right)^{2}+|X|\right) \frac{X}{|X|} & \text { for } X \in \Omega \backslash B\left(0, \frac{R_{1}}{4}\right) .\end{cases}
$$

Note that $\Phi$ is a $C^{1, \alpha}$ map. For $Y \in B\left(0, R_{1}\right) \subset \Omega$ there exists a unique $Q_{Y} \in \partial \Omega$. Since $g$ is a bijection there exists a unique $t \in\left[0,\left|Q_{Y}\right|\right]$ so that $|Y|=g(t)$. Since $\Omega$ is star-shaped with respect to the origin there exists $X \in \Omega$, such that $X=t \frac{Q_{Y}}{\left|Q_{Y}\right|}$. This implies that $\Phi(X)=Y$. If $\Phi(X)=\Phi\left(X^{\prime}\right) \Rightarrow g(|X|)=g\left(\left|X^{\prime}\right|\right)$ and $\frac{X}{|X|}=\frac{X^{\prime}}{\left|X^{\prime}\right|}$. Since $g$ is $1-1$, $|X|=\left|X^{\prime}\right|$ which yields $X=X^{\prime}$. Thus $\Phi: \Omega \rightarrow B\left(0, R_{1}\right)$ is a $C^{1, \alpha}$ bijection. It is easy to check that $\Phi^{-1}$ is also $C^{1, \alpha}$.

Lemma 5.2 Assume that $\Omega \subset \mathbb{R}^{n+1}$ satisfies (2.9). Given $\delta>0$ there exists $\varepsilon>0$ such that if $\sup _{\partial \Omega}|\log h|<\varepsilon$ with $\varepsilon<\varepsilon_{0}$ then there exists $\rho_{\varepsilon}>0$ such that for $r \in\left(0, \rho_{\varepsilon}\right)$ and $Q \in \partial \Omega$

$$
\frac{H^{n}(B(Q, r) \cap \partial \Omega)}{\omega_{n} r^{n}} \leq(1+\delta)
$$

Proof. Let $\varphi \in C_{c}^{\infty}\left(\mathbb{R}^{n+1}\right)$ such that $0 \notin \operatorname{supp} \varphi$ then

$$
-\int_{\Omega}\langle\nabla \varphi, \nabla G\rangle=\int_{\partial \Omega} \varphi h d \sigma .
$$


By choosing $\varphi$ as an approximation of $\chi_{B(Q, r)}$ we obtain after passing to the limit that for a.e. $r>0$ with $r<\frac{R_{1}}{4}$

$$
\int_{\partial \Omega \cap B(Q, r)} h d \mathcal{H}^{n}=\int_{\partial B(Q, r) \cap \Omega}\left\langle\nabla G, \frac{X-Q}{|X-Q|}\right\rangle d \mathcal{H}^{n} .
$$

For the details of this computation see [9] section 3 .

Let $\delta^{\prime}=\delta^{\prime}(\delta) \in(0,1)$ and choose $\varepsilon_{0}^{\prime} \in\left(0, \frac{1}{4}\right)$ so that if $\sup |\log h|<\varepsilon^{\prime}$ for $\varepsilon^{\prime} \in\left(0, \varepsilon_{0}^{\prime}\right)$ then $\Omega$ is a $\delta^{\prime}$-Reifenberg flat chord arc domain (see Corollary 5.1) and

$$
G \in F\left(\frac{\delta^{\prime}}{2}, \frac{\delta^{\prime}}{2},\left(e^{2 \varepsilon^{\prime}-1}\right)^{1 / 4}\right) \quad \text { in } \quad B\left(Q,\left(\frac{\eta}{2}\right)^{k} \rho^{\prime}\right)
$$

for all $Q \in \partial \Omega, k \geq 1$ where $\rho^{\prime}=\sqrt{2} \sqrt{e^{2 \varepsilon^{\prime}-1}} R_{1}$ and $\eta \in\left(0, \frac{1}{4}\right)$ (see Lemma 4.3 and the proof of Theorem 2.1, namely (4.28) and (4.29)).

By Lemma 3.3, $B\left(0, R_{1}\right) \subset \Omega \subset B\left(0, R_{2}\right)$ with $e^{-\varepsilon^{\prime}} \leq \sigma_{n} R_{1}^{n} \leq \sigma_{n} R_{2}^{n} \leq$ $e^{\varepsilon^{\prime}}$. Note that since $G \in F\left(\frac{\delta^{\prime}}{2}, \frac{\delta^{\prime}}{2},\left(e^{2 \varepsilon^{\prime}}-1\right)^{1 / 4}\right)$ in $B\left(Q,\left(\frac{\eta}{2}\right)^{k} \rho^{\prime}\right)$ for $k \geq 1$ then $G \in F\left(\delta^{\prime}, \delta^{\prime},\left(e^{2 \varepsilon^{\prime}}-1\right)^{1 / 4}\right)$ in $B(Q, r)$ for $r \in\left(0, \rho^{\prime}\right)$. Thus there exists $\overrightarrow{n_{Q, r}} \in \mathbb{S}^{n}$ so that

$$
G(X)=0 \quad \text { for } \quad\left\langle X-Q ; \overrightarrow{n_{Q, r}}\right\rangle \leq-\delta^{\prime} r
$$

and

$$
G(X) \geq h(Q)\left[\left\langle X-Q ; \overrightarrow{n_{Q, r}}\right\rangle-\delta^{\prime} r\right] \text { for }\left\langle X-Q ; \overrightarrow{n_{Q, r}}\right\rangle \geq \delta^{\prime} r .
$$

To estimate the term in the right hand side of (5.13) consider

$$
\begin{aligned}
0 \leq & \int_{\partial B(Q, r) \cap \Omega}\left\langle\nabla G ; \frac{X-Q}{|X-Q|}\right\rangle d \mathcal{H}^{n} \\
\leq & \int_{\partial B(Q, r) \cap\left\{x+t \overrightarrow{n_{Q, r}}: t \geq 2 \sqrt{\delta^{\prime} r}\right\}}\left\langle\nabla G(X) ; \frac{X-Q}{|X-Q|}\right\rangle d \mathcal{H}^{n} \\
& \quad+\int_{\partial B(Q, r) \cap\left\{x+t \overrightarrow{n_{Q, r}}:-\delta^{\prime} r \leq t \leq 2 \sqrt{\delta^{\prime}} r\right\}}|\nabla G| d \mathcal{H}^{n}
\end{aligned}
$$

Here the decomposition $x+t \overrightarrow{n_{Q, r}}$ means that $x \in L(Q, r)$ where $L(Q, r)$ is an $n$-plane through $Q$, orthogonal to $\overrightarrow{n_{Q, r}}$.

Given our choice of $r$, Lemma 4.3 guarantees that

$$
\sup _{B(Q, r)}|\nabla G| \leq h(Q)\left(1+\left(e^{2 \varepsilon^{\prime} 1}-1\right)^{1 / 4}\right) \leq h(Q)\left(1+2\left(\varepsilon^{\prime}\right)^{1 / 4}\right),
$$

for $\varepsilon_{0}^{\prime}$ small enough. 
Using (5.17) a simple computation yields

$$
\int_{\partial B(Q, r) \cap\left\{x+t \overrightarrow{n_{Q, r}} ;-\delta^{\prime} r \leq t \leq 2 \sqrt{\delta^{\prime}} r\right\}}|\nabla G| d \mathcal{H}^{n} \leq C_{n} \sqrt{\delta^{\prime}} r^{n} .
$$

Combining (5.14), (5.15) and (5.17) we have for $X \in B(Q, r), X=x+t \overrightarrow{n_{Q, r}}$ with $t \geq 2 \delta^{\prime} r \geq 2 \sqrt{\delta^{\prime}} r$

$$
h(Q)\left(t-\delta^{\prime} r\right) \leq G(X) \leq h(Q)\left(1+2\left(\varepsilon^{\prime}\right)^{1 / 4}\right)\left(t+\delta^{\prime} r\right) .
$$

Note that for such $X$, if $d(X)$ denotes the distance from $X$ to $\partial \Omega$ then

$$
r \geq d(X) \geq t-\delta^{\prime} r \geq \frac{t}{2}
$$

As in (4.22) and (4.23) we have that

$$
\begin{aligned}
\nabla G(X) & =\frac{-2^{n+2}}{\omega_{n+1} d(X)^{n+2}} \int_{\partial B\left(X, \frac{d(X)}{2}\right)} G(\zeta)(X-\zeta) d \zeta \\
& =-\frac{2^{n+2}}{\omega_{n+1} d(X)^{n+2}} \int_{\partial B\left(X, \frac{d(X)}{2}\right)}\left(G(\zeta)-h(Q) \widetilde{t}_{\zeta}\right)(X-\zeta) d \zeta \\
& -\frac{2^{n+2}}{\omega_{n+1} d(X)^{n+2}} \int_{\partial B\left(X, \frac{d(X)}{2}\right)} h(Q) \widetilde{t}_{\zeta}(X-\zeta) d \zeta
\end{aligned}
$$

where $\widetilde{t}_{\zeta}=\left\langle\zeta-Q, \vec{n}_{Q, 2 r}\right\rangle$. Note that if $\zeta \in \partial B\left(X, \frac{d(X)}{2}\right)$, then $\zeta \in B(Q, 2 r)$. The first equality in (5.21) applied to the function $\widetilde{t}_{\zeta}$ rather than $G$ guarantees that

$$
\frac{2^{n+2}}{\omega_{n+1} d(X)^{n+2}} \int_{\partial B\left(X, \frac{d(X)}{2}\right)} h(Q) \widetilde{t}_{\zeta}(X-\zeta) d \zeta=h(Q) \vec{n}_{Q, 2 r} .
$$

Since $\left|\widetilde{t}_{\zeta}\right| \leq 2 r$ using (5.19) we have that

$$
\begin{aligned}
\left|\nabla G(X)-h(Q) \vec{n}_{Q, 2 r}\right| & \leq \frac{C_{n}}{d(X)^{n+1}} \int_{\partial B\left(X, \frac{d(X)}{2}\right)}\left|G(\zeta)-h(Q) \widetilde{t}_{\zeta}\right| d \zeta \\
& \leq \frac{C_{n} h(Q)}{d(X)^{n+1}} \int_{\partial B\left(X, \frac{d(X)}{2}\right)}\left(\left(\varepsilon^{\prime}\right)^{1 / 4}\left(\left|\widetilde{t}_{\zeta}\right|+\delta^{\prime} r\right)+\delta^{\prime} r\right) d \zeta \\
& \leq \frac{C_{n} h(Q)}{d(X)}\left(\left(\varepsilon^{\prime}\right)^{1 / 4} r+\delta^{\prime} r\right) \leq C_{n} \frac{h(Q)}{t}\left(\left(\varepsilon^{\prime}\right)^{1 / 4} r+\delta^{\prime} r\right) .
\end{aligned}
$$


Using (5.20) and (5.23) we can estimate the remaining term in (5.16). Namely

$$
\begin{aligned}
& \int_{\partial B(Q, r) \cap\left\{x+t \vec{n}_{Q, r}: t \geq 2 \sqrt{\delta^{\prime}} r\right\}}\left\langle\nabla G(X) ; \frac{X-Q}{|X-Q|}\right\rangle d \mathcal{H}^{n} \\
& \leq h(Q) \int_{\partial B(Q, r) \cap\left\{x+t \vec{n}_{Q, r}: t \geq 2 \sqrt{\delta^{\prime}} r\right\}}\left\langle\vec{n}_{Q, 2 r}, \frac{X-Q}{|X-Q|}\right\rangle d \mathcal{H}^{n} \\
&+C_{n} h(Q) \frac{\left(\varepsilon^{\prime}\right)^{1 / 4}+\delta^{\prime}}{\sqrt{\delta^{\prime}}} r^{n} .
\end{aligned}
$$

Choosing $\varepsilon_{0}^{\prime}>0$ so that $\varepsilon_{0}^{\prime} \leq\left(\delta^{\prime}\right)^{4}$, and recalling that $h(Q) \leq e^{\varepsilon^{\prime}} \leq 2$ (5.24) becomes

$$
\begin{aligned}
& \int_{\partial B(Q, r) \cap\left\{x+t \overrightarrow{n_{Q, r}}: t \geq 2 \sqrt{\delta^{\prime}} r\right\}}\left\langle\nabla G ; \frac{X-Q}{|X-Q|}\right\rangle d \mathcal{H}^{n}
\end{aligned}
$$

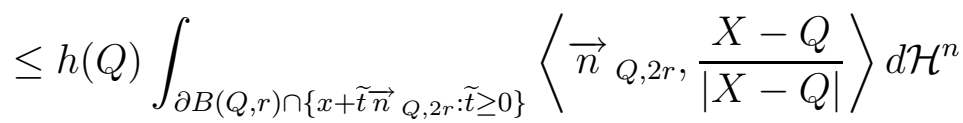

$$
\begin{aligned}
& +2 \mathcal{H}^{n}\left(\partial B(Q, r) \cap\left(\left\{x+\widetilde{t} \vec{n}_{Q, 2 r}: \widetilde{t} \geq 0\right\} \Delta\left\{x+t \vec{n}_{Q, r}: t \geq 2 \sqrt{\delta^{\prime}} r\right\}\right)\right) \\
& +C_{n} \sqrt{\delta^{\prime}} r^{n} .
\end{aligned}
$$

A simple computation shows that the angle between $\vec{n}_{Q, 2 r}$ and $\vec{n}_{Q, r}$ is less than $C \delta^{\prime}$. This fact combined with (5.13) applied to the function $\widetilde{t} \vec{n}_{Q, 2 r}$ instead of $G$ and (5.25) implies

$$
\begin{gathered}
\int_{\partial B(Q, r) \cap\left\{x+t \vec{n}_{Q, r}, t \geq 2 \sqrt{\delta^{\prime}} r\right\}}\left\langle\nabla G, \frac{X-Q}{|X-Q|}\right\rangle d \mathcal{H}^{n} \\
\leq h(Q) \int_{L(Q, 2 r) \cap B(Q, r)} d \mathcal{H}^{n}+C_{n} \sqrt{\delta^{\prime}} r^{n}
\end{gathered}
$$

Combining (5.13), (5.16), (5.18) and (5.26) plus the fact that $e^{-\varepsilon^{\prime}} \leq$ $h(P) \leq e^{\varepsilon^{\prime}}$ for $P \in \partial \Omega$ we conclude for $r \leq \sqrt{2} \sqrt{e^{2 \varepsilon^{\prime}}-1} R_{1}$

$$
\begin{aligned}
\mathcal{H}^{n}(B(Q, r) \cap \partial \Omega) & =\int_{B(Q, r) \cap \partial \Omega} h(P) h^{-1}(P) d \mathcal{H}^{n} \\
& \leq e^{\varepsilon^{\prime}} \int_{B(Q, r) \cap \partial \Omega} h d \mathcal{H}^{n} \leq e^{2 \varepsilon^{\prime}} \omega_{n} r^{n}+C_{n} \sqrt{\delta^{\prime}} r^{n} .
\end{aligned}
$$

By our choice of $\varepsilon_{0}^{\prime}>0$ (so that $\left.\varepsilon_{0}^{\prime} \leq\left(\delta^{\prime}\right)^{4}\right)$ we have that for $r \leq$ $\sqrt{2} \sqrt{e^{2 \varepsilon^{\prime}}-1} R_{1}$

$$
\mathcal{H}^{n}(B(Q, r) \cap \partial \Omega) \leq \omega_{n} r^{n}\left(1+C_{n} \sqrt{\delta^{\prime}}\right) .
$$

Choosing $\delta^{\prime}>0$ so that $C_{n}\left(\delta^{\prime}\right)^{1 / 2}=\delta$, and $\varepsilon_{0}$ the corresponding $\varepsilon_{0}^{\prime}$ we have proved the statement of Lemma 5.2. 
Corollary 5.4 Assume that $\Omega \subset \mathbb{R}^{n+1}$ satisfies (2.9). Given $\delta>0$ there exists $\varepsilon>0$ such that if $\sup _{\partial \Omega}|\log h|<\varepsilon$ then $\Omega$ is a $\delta$-chord arc domain.

Proof. From the proof of Lemma 5.1 (see (5.3)) and Lemma 5.2 we have that given $\delta>0$ there exist $\varepsilon>0$ and $\rho>0$ so that if $\sup _{\partial \Omega}|\log h|<\varepsilon$ then for $r \in(0, \rho)$ and $Q \in \partial \Omega$

$$
(1+\delta)^{-1} \leq \frac{H^{n}(\partial \Omega \cap B(Q, r))}{\omega_{n} r^{n}} \leq 1+\delta .
$$

By Theorem 2.1 we also know that $\rho>0$ can be chosen so that

$$
\theta(Q, \rho) \leq \delta
$$

This is a straightforward consequence of Corollary 4.5 (for the proof, see $[7, \S 2])$.

Corollary 5.5 Assume that $\Omega \subset \mathbb{R}^{n+1}$ satisfies (2.9). Given $\delta>0$ there exist $\varepsilon>0$ and $\rho>0$ such that if $\sup _{\partial \Omega}|\log h|<\varepsilon$ then

$$
\|\vec{n}\|_{*}(\rho)=\sup _{Q \in \partial \Omega} \sup _{0<r<\rho}\left(f_{B(Q, r) \cap \partial \Omega}\left|\vec{n}-\vec{n}{ }_{Q, r}\right|^{2} d \sigma\right)^{1 / 2} \leq \delta .
$$

\section{References}

[1] Alt, H. W. and Caffarelli, L. A.: Existence and regularity for a minimum problem with free boundary. J. Reine Angew. Math. 325 (1981), 105-144.

[2] Evans, L. C. And Gariepy, R. F.: Measure theory and fine properties of functions. Studies in Advanced Mathematics. CRC Press, Boca Raton, FL, 1992.

[3] Gilbarg, D. and Trudinger, N. S.: Elliptic partial differential equations of second order. Fundamental Principles of Mathematical Sciences, 224. Springer-Verlag, Berlin, 1983.

[4] Helms, L. L.: Introduction to potential theory. Pure and Applied Mathematics 23. Robert E. Krieger Publishing Co., Huntington, N.Y., 1975.

[5] Jerison, D. And Kenig, C.: Boundary behavior of harmonic functions in nontangentially accessible domains. Adv. in Math. 46 (1982), 80-147.

[6] Kenig, C. And Toro, T.: Free boundary regularity for harmonic measures and poisson kernels. Ann. of Math. (2) 150 (1999), 369-454.

[7] Kenig, C. And Toro, T.: Harmonic measure on locally flat domains. Duke Math. J. 87 (1997), 509-551. 
[8] Kenig, C. And Toro, T.: Poisson kernel characterization of Reifenberg flat chord arc domains. Ann. Sci. École Norm. Sup. (4) 36 (2003), 323-401.

[9] Kenig, C. And Toro, T.: Free boundary regularity below the continuous threshold: 2-phase problems. J. Reine Angew. Math. 596 (2006), 1-44.

[10] Lewis, J. And Vogel, A.: A symmetry theorem revisited. Proc. Amer. Math. Soc. 130 (2002), no. 2, 443-451.

[11] Lewis, J. AND Vogel, A.: On some almost everywhere symmetry theorems. In Nonlinear diffusion equations and their equilibrium states, 3 (Gregynog, 1989), 347-374. Progr. Nonlinear Differential Equations Appl. 7. Birkhäuser Boston, Boston, MA, 1992.

Recibido: 19 de octubre de 2004

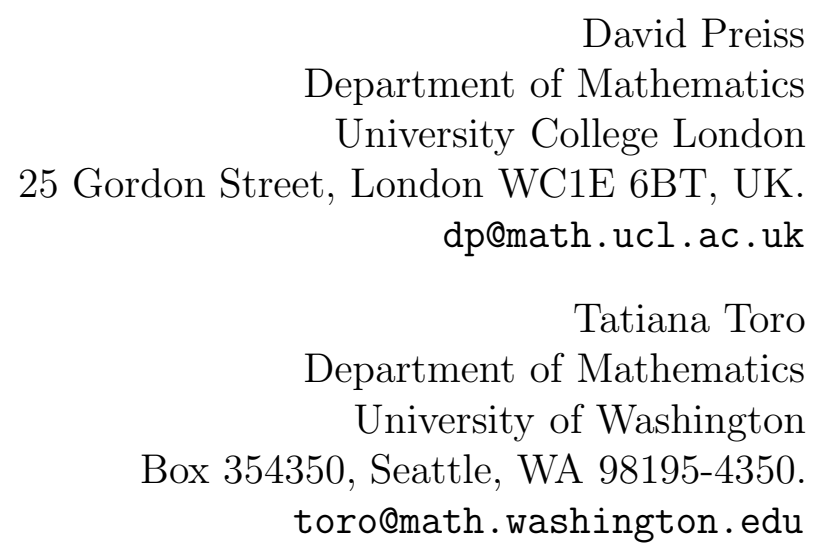

The second author was partially supported by NSF through DMS and by a Transitional Support Grant from ADVANCE at UW. 